Supporting Information 


\title{
Sulfide Perovskites for Thermoelectricity
}

\author{
Hatef Shahmohamadi ${ }^{1}$ and S. Shahab Naghavi ${ }^{1 *}$ \\ ${ }^{1}$ Shahid Beheshti University, Department of Physical and Computational Chemistry, Shahid \\ Beheshti University, Evin, 1983969411 Tehran, Iran \\ E-mail: s_naghavi.sbu.ac.ir
}


Table S1: Compounds with a bold font are predicted compounds and the rest are from ICSD.

\begin{tabular}{|c|c|c|c|c|c|c|c|c|c|c|c|c|c|c|c|c|c|}
\hline No. & Material & Symm. & Prototype & Egap & Direct & Pfxx(hole) & Pfyy(hole) & Pfzz(hole) & Pfxx(elec) & Pfyy(elec) & Pfzz(elec) & $a$ & $b$ & $c$ & $\alpha$ & $\beta$ & $\gamma$ \\
\hline 1 & $\mathrm{SbGaS}_{3}$ & Pnma (62) & $\mathrm{NH}_{4} \mathrm{CdCl} 3 / \mathrm{Sn}_{2} \mathrm{~S}_{3}$ & 1.6 & $\mathrm{~F}$ & 4.73 & 0.38 & 0.17 & 2.35 & 0.31 & 0.33 & 3.89 & 9.17 & 14.879 & 90.09 & 90.0 & 90.0 \\
\hline 2 & $\mathrm{TaNaS}_{3}$ & $\mathrm{C} 2 / \mathrm{m}(12)$ & $\mathrm{FePS}_{3}$ & 1.4 & F & 3.44 & 3.98 & 2.47 & 2.17 & 3.48 & 1.44 & 11.13 & 6.50 & 7.32 & 65.29 & 90.0 & 90.0 \\
\hline 3 & $\mathrm{CuTaS}_{3}$ & Pnma (62) & $\mathrm{CuTaS}_{3}$ & 0.4 & $\mathrm{~T}$ & 3.38 & 1.64 & 0.27 & 3.13 & 0.5 & 0.09 & 3.52 & 9.61 & $11.92 \mathrm{~s}$ & 90.09 & 90.0 & 90.0 \\
\hline 4 & $\mathrm{TaLiS}_{3}$ & $\mathrm{C} 2 / \mathrm{m}(12)$ & $\mathrm{FePS}_{3}$ & 1.0 & F & 3.35 & 2.37 & 2.07 & 2.43 & 3.16 & 0.95 & 10.83 & 6.32 & 6.79 & 65.89 & 90.0 & 90.0 \\
\hline 5 & $\mathrm{BaSnS}_{3}$ & Pnma (62) & $\mathrm{NH}_{4} \mathrm{CdCl} 3 / \mathrm{Sn}_{2} \mathrm{~S}_{3}$ & 0.8 & $\mathrm{~F}$ & 3.19 & 1.3 & 0.05 & 0.48 & 2.43 & 0.2 & 3.99 & 8.61 & $14.80 \mathrm{~s}$ & 90.09 & 90.0 & 90.0 \\
\hline 6 & $\mathrm{InSbS}_{3}$ & Pnma (62) & $\mathrm{NH}_{4} \mathrm{CdCl} 3 / \mathrm{Sn}_{2} \mathrm{~S}_{3}$ & 1.5 & $\mathrm{~F}$ & 3.18 & 1.05 & 2.96 & 1.56 & 0.37 & 0.3 & 3.90 & 9.50 & $13.60 \mathrm{c}$ & 90.09 & 90.0 & 90.0 \\
\hline 7 & $\mathrm{BaBiSe}_{3}$ & P212121 (19) & $\mathrm{BaBiSe}_{3}$ & 0.7 & F & 3.01 & 0.34 & 1.64 & 0.56 & 0.02 & 1.29 & 4.43 & 16.57 & $17.16 \mathrm{~s}$ & 90.09 & 90.0 & 90.0 \\
\hline 8 & $\mathrm{BaZrS}_{3}$ & Pnma (62) & $\mathrm{GdFeO}_{3}$ & 1.0 & $\mathrm{~T}$ & 2.59 & 2.45 & 2.96 & 2.07 & 2.37 & 2.59 & 7.04 & 7.16 & $10.04 \mathrm{~s}$ & 90.09 & 90.0 & 90.0 \\
\hline 9 & $\mathrm{BaHfS}_{3}$ & Pnma (62) & $\mathrm{GdFeO}_{3}$ & 1.3 & $\mathrm{~T}$ & 2.5 & 2.44 & 2.97 & 2.05 & 2.32 & 2.18 & 7.03 & 7.10 & 9.98 & 90.09 & 90.0 & 90.0 \\
\hline 10 & $\mathrm{SrSnS}_{3}$ & Pnma (62) & $\mathrm{NH}_{4} \mathrm{CdCl} 3 / \mathrm{Sn}_{2} \mathrm{~S}_{3}$ & 0.7 & $\mathrm{~F}$ & 2.45 & 1.92 & 0.04 & 2.23 & 0.45 & 0.25 & 3.92 & 8.32 & $14.31 \mathrm{~s}-\mathrm{cos}$ & 90.09 & 90.0 & 90.0 \\
\hline 11 & $\mathrm{CaSnS}_{3}$ & Pnma (62) & $\mathrm{NH}_{4} \mathrm{CdCl} 3 / \mathrm{Sn}_{2} \mathrm{~S}_{3}$ & 0.2 & $\mathrm{~T}$ & 2.17 & 0.06 & 0.1 & 0.67 & 0.53 & 1.28 & 3.88 & 10.64 & $11.27 \mathrm{~g}$ & 90.09 & 90.0 & 90.0 \\
\hline 12 & $\mathrm{PbHfS}_{3}$ & Pnma (62) & $\mathrm{NH}_{4} \mathrm{CdCl} 3 / \mathrm{Sn}_{2} \mathrm{~S}_{3}$ & 1.0 & $\mathrm{~T}$ & 1.97 & 1.97 & 2.5 & 1.41 & 1.41 & 5.05 & 3.78 & 9.26 & 14.169 & 90.09 & 90.0 & 90.0 \\
\hline 13 & $\mathrm{BaTeS}_{3}$ & Pnma (62) & ragonite- $-\mathrm{CaCO}_{3}(\mathrm{OP} 20)$ & 1.8 & $\mathrm{~F}$ & 1.96 & 1.88 & 2.63 & 1.54 & 0.72 & 0.68 & 7.02 & 7.41 & $11.22 \mathrm{~g}-\mathrm{a}$ & 90.09 & 90.0 & 90.0 \\
\hline 14 & $\mathrm{SbYS}_{3}$ & Pnma (62) & $\mathrm{NH}_{4} \mathrm{CdCl} 3 / \mathrm{Sn}_{2} \mathrm{~S}_{3}$ & 1.4 & $\mathrm{~F}$ & 1.83 & 0.61 & 0.79 & 2.12 & 0.28 & 0.1 & 3.95 & 10.86 & 12.16 & 90.09 & 90.0 & 90.0 \\
\hline 15 & $\mathrm{TiGeS}_{3}$ & Pnma (62) & $\mathrm{NH}_{4} \mathrm{CdCl} 3 / \mathrm{Sn}_{2} \mathrm{~S}_{3}$ & 0.2 & $\mathrm{~T}$ & 1.82 & 0.67 & 0.2 & 3.75 & 0.24 & 0.02 & 3.49 & 9.29 & 13.839 & 90.09 & 90.0 & 90.0 \\
\hline 16 & $\mathrm{CsAuSe}_{3}$ & $\mathrm{C} 12 / \mathrm{cl}(15)$ & $\mathrm{CsAuSe}_{3}$ & 1.1 & F & 1.81 & 1.08 & 1.91 & 0.15 & 0.6 & 0.7 & 13.97 & 6.76 & 7.80 & 69.69 & 90.0 & 90.0 \\
\hline 17 & $\mathrm{GaYS}_{3}$ & Pnma (62) & $\mathrm{NH}_{4} \mathrm{CdCl} 3 / \mathrm{Sn}_{2} \mathrm{~S}_{3}$ & 0.8 & $\mathrm{~T}$ & 1.65 & 3.15 & 0.18 & 0.44 & 0.12 & 0.47 & 3.76 & 8.28 & $12.61 \mathrm{~s}$ & 90.09 & 90.0 & 90.0 \\
\hline 18 & $\mathrm{TiCdS}_{3}$ & $\mathrm{C} 2 / \mathrm{m}(13)$ & $\mathrm{FePS}_{3}$ & 0.5 & $\mathrm{~T}$ & 1.59 & 0.88 & 1.47 & 0.82 & 1.09 & 0.91 & 11.09 & 6.43 & 6.816 & 64.09 & 90.0 & 90.0 \\
\hline 19 & $\mathrm{ScSbS}_{3}$ & Pnma (62) & $\mathrm{NH}_{4} \mathrm{CdCl} 3 / \mathrm{Sn}_{2} \mathrm{~S}_{3}$ & 1.3 & F & 1.53 & 0.34 & 0.6 & 3.08 & 0.06 & 0.28 & 3.78 & 9.50 & $13.24 \mathrm{~s}$ & 90.09 & 90.0 & 90.0 \\
\hline 20 & $\mathrm{SrTeS}_{3}$ & Pna21 (33) & Perovskite-ScYS & 0.7 & $\mathrm{~F}$ & 1.41 & 2.75 & 2.15 & 1.19 & 4.29 & 2.96 & 3.99 & 11.05 & $11.57 \mathrm{~g}$ & 90.09 & 90.0 & 90.0 \\
\hline 21 & $\mathrm{TaTlSe}_{3}$ & Pnma (62) & $\mathrm{NH}_{4} \mathrm{CdCl} 3 / \mathrm{Sn}_{2} \mathrm{~S}_{3}$ & 0.3 & $\mathrm{~T}$ & 1.39 & 4.78 & 0.23 & 0.21 & 3.58 & 0.15 & 3.65 & 9.79 & $15.20 \mathrm{~s}$ & 90.09 & 90.0 & 90.0 \\
\hline 22 & BiTIS $_{3}$ & $\mathrm{C} 2 / \mathrm{m}(12)$ & $\mathrm{FePS}_{3}$ & 0.4 & $\mathrm{~F}$ & 1.34 & 1.7 & 2.87 & 0.46 & 0.53 & 0.68 & 12.07 & 7.15 & 7.166 & 68.29 & 90.0 & 90.0 \\
\hline 23 & $\mathrm{CrSbS}_{3}$ & Pnma (62) & $\mathrm{NH}_{4} \mathrm{CdCl} 3 / \mathrm{Sn}_{2} \mathrm{~S}_{3}$ & 1.2 & F & 1.29 & 0.63 & 0.53 & 1.34 & 1.47 & 0.37 & 3.66 & 8.87 & $12.83 \mathrm{~s}$ & 90.09 & 90.0 & 90.0 \\
\hline 24 & $\mathrm{GaScS}_{3}$ & Pnma (62) & $\mathrm{NH}_{4} \mathrm{CdCl} 3 / \mathrm{Sn}_{2} \mathrm{~S}_{3}$ & 1.7 & $\mathrm{~F}$ & 1.22 & 0.64 & 0.81 & 2.27 & 0.79 & 0.01 & 3.70 & 10.93 & $12.49 \mathrm{~s}$ & 90.09 & 90.0 & 90.0 \\
\hline 25 & $\mathrm{PbSnS}_{3}$ & Pnma (62) & $\mathrm{NH}_{4} \mathrm{CdCl} 3 / \mathrm{Sn}_{2} \mathrm{~S}_{3}$ & 1.0 & $\mathrm{~T}$ & 1.17 & 1.02 & 0.26 & 0.71 & 3.01 & 1.39 & 3.86 & 9.02 & $14.27 \mathrm{~s}$ & 90.09 & 90.0 & 90.0 \\
\hline 26 & $\mathrm{SrBiSe}_{3}$ & P212121 (19) & $\mathrm{SrBiSe}_{3}$ & 0.4 & F & 1.13 & 0.79 & 0.65 & 0.02 & 0.01 & 2.01 & 4.27 & 16.12 & $33.79 \mathrm{~s}$ & 90.09 & 90.0 & 90.0 \\
\hline 27 & $\mathrm{SrSbSe}_{3}$ & P212121 (19) & $\mathrm{SrBiSe}_{3}$ & 0.4 & $\mathrm{~F}$ & 1.13 & 0.37 & 1.06 & 0.03 & 0.04 & 1.89 & 4.27 & 15.87 & 33.559 & 90.09 & 90.0 & 90.0 \\
\hline 28 & $\mathrm{AlInS}_{3}$ & P61 (169) & $\mathrm{Al}_{2} \mathrm{~S}_{3}$ & 2.2 & $\mathrm{~F}$ & 1.13 & 1.13 & 1.83 & 0.63 & 0.63 & 0.95 & 6.86 & 6.86 & $18.10 \mathrm{~s}$ & 90.09 & 90.01 & 120.0 \\
\hline 29 & $\mathrm{SrHfS}_{3}$ & Pnma (62) & $\mathrm{GdFeO}_{3}$ & 1.5 & $\mathrm{~T}$ & 1.02 & 1.24 & 0.74 & 1.72 & 2.19 & 2.88 & 6.76 & 7.11 & 9.77 & 90.09 & 90.0 & 90.0 \\
\hline 30 & $\mathrm{SnZrS}_{3}$ & Pnma (62) & $\mathrm{NH}_{4} \mathrm{CdCl} 3 / \mathrm{Sn}_{2} \mathrm{~S}_{3}$ & 1.0 & $\mathrm{~F}$ & 1 & 0.8 & 0.26 & 4.39 & 0.07 & 0.06 & 3.75 & 9.53 & 14.119 & 90.09 & 90.0 & 90.0 \\
\hline 31 & $\mathrm{CaZrS}_{3}$ & Pnma (62) & $\mathrm{GdFeO}_{3}$ & 1.2 & $\mathrm{~T}$ & 0.99 & 1.4 & 1.05 & 2.31 & 3.53 & 2.33 & 6.57 & 7.07 & 9.64 & 90.09 & 90.0 & 90.0 \\
\hline 32 & $\mathrm{CaZrSe}_{3}$ & Pnma (62) & $\mathrm{GdFeO}_{3}$ & 0.8 & $\mathrm{~T}$ & 1.12 & 0.59 & 2.04 & 2.31 & 3.33 & 2.07 & 6.82 & 7.38 & $10.05 \mathrm{~s}$ & 90.09 & 90.0 & 90.0 \\
\hline 33 & $\mathrm{LaLuS}_{3}$ & Pna21 (33) & Perovskite-ScYS & 2.2 & $\mathrm{~T}$ & 0.98 & 0.75 & 0.73 & 1.42 & 3.21 & 0.68 & 6.62 & 7.43 & 9.98 & 90.09 & 90.0 & 90.0 \\
\hline 34 & $\mathrm{CaHfS}_{3}$ & Pnma (62) & $\mathrm{GdFeO}_{3}$ & 1.5 & $\mathrm{~T}$ & 0.95 & 1.32 & 1.34 & 2.15 & 3.37 & 2.37 & 6.56 & 7.02 & 9.59 & 90.09 & 90.0 & 90.0 \\
\hline 35 & $\mathrm{SbCrSe}_{3}$ & Pnma (62) & $\mathrm{NH}_{4} \mathrm{CdCl} 3 / \mathrm{Sn}_{2} \mathrm{~S}_{3}$ & 1.1 & F & 0.91 & 1.16 & 0.25 & 1.54 & 1.57 & 0.7 & 3.65 & 8.52 & $14.80 \mathrm{~s}$ & 90.09 & 90.0 & 90.0 \\
\hline 36 & $\mathrm{GaInS}_{3}$ & P61 (169) & $\mathrm{Al}_{2} \mathrm{~S}_{3}$ & 1.9 & F & 0.91 & 0.91 & 0.85 & 0.2 & 0.2 & 0.24 & 6.87 & 6.87 & $18.31 \mathrm{~s}$ & 90.09 & 90.01 & 120.0 \\
\hline 37 & $\mathrm{SnHfS}_{3}$ & Pnma (62) & $\mathrm{NH}_{4} \mathrm{CdCl} 3 / \mathrm{Sn}_{2} \mathrm{~S}_{3}$ & 1.2 & $\mathrm{~T}$ & 0.87 & 0.85 & 0.28 & 5.18 & 0.03 & 0.05 & 3.72 & 9.48 & $14.19 \mathrm{~s}$ & 90.09 & 90.0 & 90.0 \\
\hline 38 & $\mathrm{PdGeS}_{3}$ & $\mathrm{C} 12 / \mathrm{ml}(12)$ & Dy3Ni2 & 1.3 & $\mathrm{~F}$ & 0.86 & 1.49 & 0.31 & 0.16 & 2.96 & 0.78 & 3.50 & 15.27 & 9.587 & 72.29 & 90.0 & 90.0 \\
\hline 39 & $\mathrm{GaInSe}_{3}$ & P61 (169) & $\mathrm{Al}_{2} \mathrm{~S}_{3}$ & 1.2 & $\mathrm{~T}$ & 0.81 & 0.81 & 1.14 & 0.16 & 0.16 & 0.16 & 7.16 & 7.16 & $19.25 \mathrm{~s}$ & 90.09 & 90.01 & 120.0 \\
\hline 40 & $\mathrm{PbZrS}_{3}$ & Pnma (62) & $\mathrm{NH}_{4} \mathrm{CdCl} 3 / \mathrm{Sn}_{2} \mathrm{~S}_{3}$ & 1.2 & $\mathrm{~T}$ & 0.79 & 2.03 & 0.18 & 0.14 & 3.5 & 0.08 & 3.81 & 9.26 & $14.03 \mathrm{~s}$ & 90.09 & 90.0 & 90.0 \\
\hline 41 & $\mathrm{YScS}_{3}$ & $21(33)$ & Perovskite-ScYS ${ }_{3}$ & 1.7 & F & 0.79 & 0.58 & 0.82 & 3.25 & 1.83 & 0.94 & 6.38 & 7.01 & 9.519 & 90.09 & 90.0 & 90.0 \\
\hline 42 & $\mathrm{TITaS}_{3}$ & Pnma (62) & $\mathrm{NH}_{4} \mathrm{CdCl} 3 / \mathrm{Sn}_{2} \mathrm{~S}_{3}$ & 0.6 & $\mathrm{~T}$ & 0.72 & 1.65 & 0.29 & 3.59 & 0.14 & 0.09 & 3.53 & 9.42 & $14.69 \mathrm{~s}$ & 90.09 & 90.0 & 90.0 \\
\hline 43 & $\mathrm{AlInSe}_{3}$ & P212121 (19) & $\mathrm{SrBiSe}_{3}$ & 1.5 & F & 0.72 & 0.72 & 2.13 & 0.59 & 0.59 & 0.69 & 7.17 & 7.17 & 19.069 & 90.09 & 90.01 & 120.0 \\
\hline 44 & $\mathrm{CsGaSe}_{3}$ & $\mathrm{P} 121 / \mathrm{cl}$ (14) & $\mathrm{RbBSe}_{3}$ & 1.6 & $\mathrm{~F}$ & 0.71 & 0.32 & 0.53 & 0.77 & 0.2 & 0.91 & 13.30 & 6.93 & 7.937 & 74.79 & 90.0 & 90.0 \\
\hline 45 & $\mathrm{CsGaS}_{3}$ & $\mathrm{P} 121 / \mathrm{cl}$ (14) & $\mathrm{RbBSe}_{3}$ & 2.4 & $\mathrm{~F}$ & 0.65 & 0.2 & 0.86 & 0.77 & 0.25 & 0.83 & 12.73 & 6.59 & 7.847 & 72.39 & 90.0 & 90.0 \\
\hline 46 & $\mathbf{B i G a S}_{3}$ & $\mathrm{C} 2 / \mathrm{m}$ (12) & $\mathrm{FePS}_{3}$ & 1.0 & F & 0.64 & 0.88 & 0.96 & 0.38 & 0.46 & 0.62 & 11.51 & 7.03 & 6.717 & 70.29 & 90.0 & 90.0 \\
\hline 47 & $\mathrm{GdScS}_{3}$ & Pnma (62) & $\mathrm{GdFeO}_{3}$ & 1.1 & $\mathrm{~F}$ & 0.59 & 0.85 & 0.83 & 0.36 & 0.34 & 0.35 & 6.38 & 7.04 & 9.469 & 90.09 & 90.0 & 90.0 \\
\hline 48 & $\mathrm{BaZrSe}_{3}$ & P63/mmc (194) & $\mathrm{BaNiO}_{3}$ & 0.3 & $\mathrm{~T}$ & 0.56 & 0.69 & 0.09 & 0.97 & 0.31 & 0.16 & 4.07 & 9.18 & $15.14 \mathrm{~g}$ & 90.09 & 90.0 & 90.0 \\
\hline 49 & $\mathrm{SrZrSe}_{3}$ & Pnma (62) & $\mathrm{NH}_{4} \mathrm{CdCl} 3 / \mathrm{Sn}_{2} \mathrm{~S}_{3}$ & 0.1 & $\mathrm{~T}$ & 0.52 & 0.38 & 0.11 & 0.29 & 0.86 & 0.16 & 3.99 & 9.04 & $14.63 \mathrm{~s}$ & 90.09 & 90.0 & 90.0 \\
\hline 50 & $\mathrm{BiScS}_{3}$ & $\mathrm{P} 21 / \mathrm{c}(14)$ & $\mathrm{PbPS}_{3}$ & 1.6 & F & 0.51 & 0.54 & 1.76 & 1.96 & 0.51 & 2.29 & 6.69 & 7.39 & 11.868 & 86.69 & 90.0 & 90.0 \\
\hline 51 & $\mathrm{SrZrS}_{3}$ & Pnma (62) & $\mathrm{GdFeO}_{3}$ & 0.6 & $\mathrm{~T}$ & 0.46 & 0.72 & 0.08 & 0.76 & 0.31 & 0.12 & 3.84 & 8.64 & $14.00 \mathrm{~s}$ & 90.09 & 90.0 & 90.0 \\
\hline 52 & $\mathrm{ZrCdS}_{3}$ & $\mathrm{C} 2 / \mathrm{m}(12)$ & $\mathrm{FePS}_{3}$ & 1.1 & $\mathrm{~F}$ & 0.34 & 0.78 & 1.69 & 1.2 & 1.34 & 0.98 & 11.46 & 6.69 & 6.956 & 64.59 & 90.0 & 90.0 \\
\hline 53 & $\mathrm{LaCrS}_{3}$ & Pnma (62) & $\mathrm{NH}_{4} \mathrm{CdCl} 3 / \mathrm{Sn}$ & 0.1 & $\mathrm{~F}$ & 0.33 & 0.9 & 0.45 & 0.21 & 0.27 & 0.16 & 3.81 & 9.26 & $14.03 \mathrm{~s}$ & 90.09 & 90.0 & 90.0 \\
\hline 54 & $\mathrm{LaGaS}_{3}$ & Pna21 (33) & $\mathrm{GaLaS}_{3}$ & 2.1 & F & 0.31 & 0.14 & 0.95 & 0.3 & 0.19 & 1.7 & 6.12 & 10.51 & $22.22 \mathrm{~g}$ & 90.09 & 90.0 & 90.0 \\
\hline 55 & $\mathrm{ZrZnS}_{3}$ & $\mathrm{C} 2 / \mathrm{m}(12)$ & & 0.9 & $\mathrm{~F}$ & 0.3 & 0.8 & 1.6 & 1.38 & 1.29 & 0.78 & 11.21 & 6.54 & 6.58 & 65.89 & 90.0 & 90.0 \\
\hline 56 & $\mathrm{TiZnS}_{3}$ & $\mathrm{C} 2 / \mathrm{m}$ (14) & $\mathrm{FePS}_{3}$ & 0.3 & $\mathrm{~T}$ & 0.29 & 0.85 & 1.37 & 1.01 & 1.17 & 0.73 & 10.83 & 6.26 & 6.436 & 65.59 & 90.0 & 90.0 \\
\hline 57 & $\mathrm{SnCdS}_{3}$ & $\mathrm{C} 2 / \mathrm{m}(12)$ & $\mathrm{Fe}$ & 0.4 & F & 0.2 & 0. & 2.58 & 0.12 & 0.13 & 0.13 & 11.53 & 6.65 & 6.78 & 66.99 & 90.0 & 90.0 \\
\hline 58 & $\mathrm{ScTIS}_{3}$ & $\mathrm{C} 2 / \mathrm{m}(12)$ & $\mathrm{FePS}_{3}$ & 0.3 & $\mathrm{~T}$ & 0.22 & 0.51 & 2.52 & 0.63 & 0.55 & 0.47 & 11.64 & 6.79 & 6.956 & 64.69 & 90.0 & 90.0 \\
\hline 59 & $\mathrm{EuHfS}_{3}$ & Pnma (62) & $\mathrm{GdFeO}_{3}$ & 1.5 & $\mathrm{~F}$ & 0.22 & 0.37 & 0.22 & 1.04 & 3.31 & 0.73 & 6.66 & 7.06 & 9.68 & 90.09 & 90.0 & 90.0 \\
\hline 60 & $\mathrm{LaScS}_{3}$ & Pna21 (33) & Perovskite-ScYS $_{3}$ & 1.0 & $\mathrm{~T}$ & 0.2 & 0.35 & 1.05 & 2.5 & 1.49 & 1.97 & 6.58 & 7.20 & 9.629 & 90.09 & 90.0 & 90.0 \\
\hline 61 & $\mathbf{L a S c S}_{3}$ & Pnma (62) & $\mathrm{NH}_{4} \mathrm{CdCl} 3 / \mathrm{Sn}_{2} \mathrm{~S}_{3}$ & 1.0 & $\mathrm{~T}$ & 0.2 & 0.3 & 1.05 & 2.5 & 1.49 & 1.97 & 6.58 & 7.20 & 9.62 & 90.09 & 90.0 & 90.0 \\
\hline 62 & $\mathrm{PbGeS}_{3}$ & $\mathrm{P} 121 / \mathrm{cl}$ (14) & $\mathrm{GePbS}_{3}$ & 1.7 & $\mathrm{~F}$ & 0.17 & 0.94 & 0.88 & 0.33 & 0.38 & 0.81 & 10.60 & 6.97 & 7.53 & 75.09 & 90.0 & 90.0 \\
\hline 63 & $\mathrm{GeSnS}_{3}$ & $\mathrm{P} 121 / \mathrm{cl}$ (14) & $\mathrm{GePbS}_{3}$ & 1.4 & F & 0.16 & 0.92 & 0.53 & 0.43 & 0.94 & 2.25 & 10.53 & 6.93 & 7.707 & 74.69 & 90.0 & 90.0 \\
\hline 64 & $\mathrm{LaYS}_{3}$ & $\mathrm{P} 121 / \mathrm{ml}$ (11) & $\mathrm{CeTmS}_{3}$ & 0.7 & $\mathrm{~T}$ & 0.12 & 0.38 & 0.41 & 0.19 & 0.38 & 0.23 & 4.02 & 11.12 & 21.76 & 78.69 & 90.0 & 90.0 \\
\hline 65 & $\mathrm{AgTaS}_{3}$ & Cmc21 (36) & Pyroxene-CaIrO ${ }_{3}$ & 0.0 & $\mathrm{~T}$ & 0.11 & 0.14 & 0.82 & 0.19 & 0.27 & 0.24 & 3.39 & 7.78 & $14.31 \mathrm{~s}$ & 90.09 & 90.0 & 90.0 \\
\hline 66 & $\mathrm{EuGaS}_{3}$ & $\mathrm{P} 121 / \mathrm{cl}(14)$ & $\mathrm{GePbS}_{3}$ & 0.4 & F & 0.04 & 0.01 & 0.08 & 0.08 & 0.02 & 0.01 & 10.49 & 10.11 & 12.718 & 81.19 & 90.0 & 90.0 \\
\hline 67 & $\mathrm{SrHfSe}_{3}$ & Pnma (62) & $\mathrm{NH}_{4} \mathrm{CdCl} 3 / \mathrm{Sn}_{2} \mathrm{~S}_{3}$ & 0.3 & $\mathrm{~T}$ & 0.35 & 0.51 & 0.09 & 0.98 & 0.26 & 0.1 & 3.96 & 9.03 & 14.60 & 0.09 & 90.0 & 90.0 \\
\hline
\end{tabular}




\begin{tabular}{|c|c|c|c|c|c|c|c|c|c|c|c|c|c|c|c|c|c|}
\hline No. & Material & Symm. & Prototype & Egap & Direct & $\operatorname{Pfxx}($ hole $)$ & Pfyy(hole) & Pfzz(hole) & $\operatorname{Pfxx}(\mathrm{elec})$ & Pfyy(elec) & Pfzz(elec) & $a$ & $b$ & $c$ & $\alpha$ & $\beta$ & $\gamma$ \\
\hline 1 & $\mathrm{BaTaS}_{3}$ & P63/mmc(194) & $\mathrm{BaNiO} 3$ & Metal & $\cdots$ & $\cdots$ & $\ldots$ & $\ldots$ & $\ldots$ & $\ldots$ & $\ldots$ & 6.93 & 6.93 & 5.73 & 90.0 & 90.0 & 120.0 \\
\hline 2 & $\mathrm{SrTiS}_{3}$ & $\mathrm{P} 63 / \mathrm{mmc}(194)$ & $\mathrm{BaNiO} 3$ & Metal & $\ldots$ & $\ldots$ & $\ldots$ & $\ldots$ & $\ldots$ & $\ldots$ & $\ldots$ & 6.62 & 6.62 & 5.79 & 90.0 & 90.0 & 120.0 \\
\hline 3 & $\mathrm{ErCeS}_{3}$ & $\mathrm{P} 121 / \mathrm{m1}(11)$ & CeTmS3 & Metal & $\ldots$ & $\ldots$ & $\ldots$ & $\ldots$ & $\ldots$ & $\ldots$ & $\ldots$ & 10.97 & 3.93 & 21.49 & 90.0 & 101.5 & 90.0 \\
\hline 4 & $\mathrm{ErScS}_{3}$ & Pna21(33) & Perovskite-ScYS3 & Metal & $\ldots$ & $\ldots$ & $\ldots$ & $\ldots$ & $\ldots$ & $\ldots$ & $\ldots$ & 6.97 & 6.33 & 9.42 & 90.0 & 90.0 & 90.0 \\
\hline 5 & $\mathrm{CeCrS}_{3}$ & Pnma(62) & $\mathrm{NH} 4 \mathrm{CdCl} 3 / \mathrm{Sn} 2 \mathrm{~S} 3$ & Metal & $\ldots$ & $\ldots$ & $\ldots$ & $\ldots$ & $\ldots$ & $\ldots$ & $\ldots$ & 3.77 & 7.78 & 12.92 & 90.0 & 90.0 & 90.0 \\
\hline 6 & $\mathrm{CeDyS}_{3}$ & Pnma(62) & $\mathrm{La} 2 \mathrm{~S} 3$ & Metal & $\ldots$ & $\ldots$ & $\ldots$ & $\ldots$ & $\ldots$ & $\ldots$ & $\ldots$ & 3.98 & 7.39 & 15.29 & 90.0 & 90.0 & 90.0 \\
\hline 7 & $\mathrm{CeErS}_{3}$ & Pnma(62) & CeErS3 & Metal & $\ldots$ & $\ldots$ & $\ldots$ & $\ldots$ & $\ldots$ & $\ldots$ & $\ldots$ & 3.95 & 16.47 & 21.17 & 90.0 & 90.0 & 90.0 \\
\hline 8 & $\mathrm{PrYbS}_{3}$ & $\mathrm{C} 2221(20)$ & NdYbS3 & Metal & $\ldots$ & $\ldots$ & $\ldots$ & $\ldots$ & $\ldots$ & $\ldots$ & $\ldots$ & 4.02 & 13.05 & 9.87 & 90.0 & 90.0 & 90.0 \\
\hline 9 & $\mathrm{NdCrS}_{3}$ & Pnma(62) & $\mathrm{NH} 4 \mathrm{CdCl} 3 / \mathrm{Sn} 2 \mathrm{~S} 3$ & Metal & $\ldots$ & $\ldots$ & $\ldots$ & $\ldots$ & $\ldots$ & $\ldots$ & $\ldots$ & 3.79 & 7.80 & 13.04 & 90.0 & 90.0 & 90.0 \\
\hline 10 & $\mathrm{NdYbS}_{3}$ & $\mathrm{~B} 2212(20)$ & $\mathrm{NdYbS3}$ & Metal & $\ldots$ & $\ldots$ & $\cdots$ & $\ldots$ & $\ldots$ & $\ldots$ & $\ldots$ & 7.21 & 7.21 & 8.82 & 90.0 & 90.0 & 148.1 \\
\hline 11 & $\mathrm{DyCeS}_{3}$ & $\mathrm{P} 121 / \mathrm{m} 1(11)$ & CeTmS3 & Metal & $\ldots$ & $\ldots$ & $\ldots$ & $\ldots$ & $\ldots$ & $\ldots$ & $\ldots$ & 11.09 & 3.98 & 11.32 & 90.0 & 108.8 & 90.0 \\
\hline 12 & $\mathrm{CeHoS}_{3}$ & $\mathrm{P} 121 / \mathrm{m} 1(11)$ & $\mathrm{CeTmS3}$ & Metal & $\ldots$ & $\ldots$ & $\ldots$ & $\ldots$ & $\ldots$ & $\ldots$ & $\ldots$ & 11.09 & 3.98 & 11.32 & 90.0 & 108.8 & 90.0 \\
\hline 13 & $\mathrm{CeLuS}_{3}$ & $\mathrm{C} 2221(20)$ & NdYbS3 & Metal & $\ldots$ & $\ldots$ & $\ldots$ & $\ldots$ & $\ldots$ & $\ldots$ & $\ldots$ & 6.61 & 6.61 & 9.52 & 90.0 & 90.0 & 145.5 \\
\hline 14 & $\mathrm{TmCeS}_{3}$ & $\mathrm{P} 1121 / \mathrm{m}(11)$ & CeTmS3 & Metal & $\ldots$ & $\ldots$ & $\ldots$ & $\ldots$ & $\ldots$ & $\ldots$ & $\ldots$ & 10.95 & 3.93 & 21.46 & 90.0 & 101.6 & 90.0 \\
\hline 15 & $\mathrm{TmLaS}_{3}$ & $\mathrm{P} 121 / \mathrm{m} 1(11)$ & CeTmS3 & Metal & $\ldots$ & $\ldots$ & $\ldots$ & $\ldots$ & $\ldots$ & $\ldots$ & $\ldots$ & 11.07 & 4.00 & 21.63 & 90.0 & 101.5 & 90.0 \\
\hline 16 & $\mathrm{CeTmS}_{3}$ & Pnma(62) & $\mathrm{CeErS} 3$ & Metal & $\ldots$ & $\ldots$ & $\ldots$ & $\ldots$ & $\ldots$ & $\ldots$ & $\ldots$ & 3.93 & 16.38 & 21.11 & 90.0 & 90.0 & 90.0 \\
\hline 17 & $\mathrm{LaYbS}_{3}$ & Pnma(62) & CeErS3 & Metal & $\ldots$ & $\ldots$ & $\ldots$ & $\ldots$ & $\ldots$ & $\ldots$ & $\ldots$ & 4.08 & 16.69 & 21.23 & 90.0 & 90.0 & 90.0 \\
\hline 18 & $\mathrm{LaYbS}_{3}$ & $\mathrm{C} 2221(20)$ & NdYbS3 & Metal & $\ldots$ & $\ldots$ & $\ldots$ & $\ldots$ & $\ldots$ & $\ldots$ & $\ldots$ & 11.24 & 4.16 & 11.52 & 90.0 & 105.2 & 90.0 \\
\hline 19 & $\mathrm{LaYbS}_{3}$ & $\operatorname{Pnam}(62)$ & Perovskite-GdFeO3 & Metal & $\ldots$ & $\ldots$ & $\ldots$ & $\ldots$ & $\ldots$ & $\ldots$ & $\ldots$ & 7.17 & 7.76 & 8.97 & 90.0 & 90.0 & 90.0 \\
\hline 20 & $\mathrm{LaYbS}_{3}$ & Pna21(33) & Perovskite-ScYS3 & Metal & $\ldots$ & $\ldots$ & $\ldots$ & $\ldots$ & $\ldots$ & $\ldots$ & $\ldots$ & 7.16 & 7.78 & 8.95 & 90.0 & 90.0 & 90.0 \\
\hline 21 & $\mathrm{LaYbS}_{3}$ & $\mathrm{Cmcm}(63)$ & ScUS3 & Metal & $\ldots$ & $\ldots$ & $\ldots$ & $\ldots$ & $\ldots$ & $\ldots$ & $\ldots$ & 4.06 & 13.96 & 8.87 & 90.0 & 90.0 & 90.0 \\
\hline 22 & $\mathrm{SmCrS}_{3}$ & Pnma(62) & $\mathrm{NH} 4 \mathrm{CdCl} 3 / \mathrm{Sn} 2 \mathrm{~S} 3$ & Metal & $\ldots$ & $\ldots$ & $\ldots$ & $\ldots$ & $\ldots$ & $\ldots$ & $\ldots$ & 3.76 & 7.80 & 12.90 & 90.0 & 90.0 & 90.0 \\
\hline 23 & $\mathrm{ErLaS}_{3}$ & $\mathrm{P} 121 / \mathrm{m} 1(11)$ & $\mathrm{CeTmS3}$ & Metal & $\ldots$ & $\ldots$ & $\ldots$ & $\ldots$ & $\ldots$ & $\ldots$ & $\ldots$ & 16.51 & 4.00 & 21.26 & 90.0 & 90.0 & 90.0 \\
\hline 24 & $\mathrm{ErPrS}_{3}$ & $\mathrm{P} 121 / \mathrm{m} 1(11)$ & CeTmS3 & Metal & $\ldots$ & $\ldots$ & $\ldots$ & $\ldots$ & $\ldots$ & $\ldots$ & $\ldots$ & 11.05 & 3.98 & 21.59 & 90.0 & 101.5 & 90.0 \\
\hline 25 & $\mathrm{PrScS}_{3}$ & Pna21(33) & Perovskite-ScYS3 & Metal & $\ldots$ & $\ldots$ & $\ldots$ & $\ldots$ & $\ldots$ & $\ldots$ & $\ldots$ & 7.11 & 6.49 & 9.53 & 90.0 & 90.0 & 90.0 \\
\hline 26 & $\operatorname{PrTmS}_{3}$ & $\mathrm{P} 121 / \mathrm{m} 1(11)$ & CeTmS3 & Metal & $\ldots$ & $\ldots$ & $\ldots$ & $\ldots$ & $\ldots$ & $\ldots$ & $\ldots$ & 11.04 & 3.97 & 21.58 & 90.0 & 101.5 & 90.0 \\
\hline 27 & $\mathrm{ScNdS}_{3}$ & Pna21(33) & Perovskite-ScYS3 & Metal & $\ldots$ & $\cdots$ & $\ldots$ & $\cdots$ & $\ldots$ & $\ldots$ & $\ldots$ & 7.11 & 6.45 & 9.50 & 90.0 & 90.0 & 90.0 \\
\hline 28 & $\mathrm{ScGdS}_{3}$ & Pna21(33) & Perovskite-ScYS3 & Metal & $\ldots$ & $\ldots$ & $\ldots$ & $\ldots$ & $\ldots$ & $\ldots$ & $\ldots$ & 7.04 & 9.46 & 6.38 & 90.0 & 90.0 & 90.0 \\
\hline 29 & $\mathrm{ScTbS}_{3}$ & Pna21(33) & Perovskite-ScYS3 & Metal & .. & $\ldots$ & $\ldots$ & $\ldots$ & $\ldots$ & $\ldots$ & $\ldots$ & 7.03 & 6.37 & 9.48 & 90.0 & 90.0 & 90.0 \\
\hline 30 & $\mathrm{DyScS}_{3}$ & Pna21(33) & Perovskite-ScYS3 & Metal & $\ldots$ & $\ldots$ & $\ldots$ & $\ldots$ & $\ldots$ & $\ldots$ & $\ldots$ & 7.03 & 6.36 & 9.45 & 90.0 & 90.0 & 90.0 \\
\hline 31 & $\mathrm{SmScS}_{3}$ & Pna21(33) & Perovskite-ScYS3 & Metal & $\ldots$ & $\ldots$ & $\ldots$ & $\ldots$ & $\ldots$ & $\ldots$ & $\ldots$ & 7.07 & 6.43 & 9.50 & 90.0 & 90.0 & 90.0 \\
\hline 32 & $\mathrm{HoLaS}_{3}$ & $\mathrm{P} 121 / \mathrm{m} 1(11)$ & CeTmS3 & Metal & $\ldots$ & $\ldots$ & $\ldots$ & $\ldots$ & $\ldots$ & $\ldots$ & $\ldots$ & 21.32 & 3.99 & 11.12 & 90.0 & 103.9 & 90.0 \\
\hline 33 & $\mathrm{ScHoS}_{3}$ & Pna21(33) & Perovskite-ScYS3 & Metal & $\ldots$ & $\ldots$ & $\ldots$ & $\ldots$ & $\ldots$ & $\ldots$ & $\ldots$ & 6.98 & 6.34 & 9.46 & 90.0 & 90.0 & 90.0 \\
\hline 34 & $\mathrm{NdLuS}_{3}$ & $\mathrm{C} 2221(20)$ & $\mathrm{NdYbS3}$ & Metal & $\ldots$ & $\ldots$ & $\ldots$ & $\ldots$ & $\ldots$ & $\ldots$ & $\ldots$ & 12.53 & 3.84 & 9.42 & 90.0 & 90.0 & 90.0 \\
\hline 35 & $\mathrm{PrLuS}_{3}$ & $\mathrm{C} 2221(20)$ & IYbS3 & Metal & ... & $\ldots$ & $\ldots$ & $\ldots$ & $\ldots$ & $\ldots$ & $\ldots$ & 12.57 & 3.79 & 9.47 & 90.0 & 90.0 & 90.0 \\
\hline 36 & $\mathrm{NdTmS}_{3}$ & $\mathrm{P} 121 / \mathrm{m} 1(11)$ & CeTmS3 & Metal & $\cdots$ & $\cdots$ & $\cdots$ & $\cdots$ & $\cdots$ & $\cdots$ & $\ldots$ & 21.23 & 3.95 & 11.04 & 90.0 & 103.2 & 90.0 \\
\hline 37 & $\mathrm{LaTmS}_{3}$ & Pnma(62) & $\mathrm{CeErS3}$ & Metal & $\ldots$ & $\ldots$ & $\ldots$ & $\ldots$ & $\ldots$ & $\ldots$ & $\ldots$ & 16.45 & 3.99 & 21.21 & 90.0 & 90.0 & 90.0 \\
\hline 38 & $\mathrm{BaVS}_{3}$ & P63/mmc(194) & $\mathrm{BaNiO} 3$ & Metal & 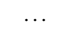 & $\ldots$ & $\ldots$ & $\ldots$ & $\ldots$ & $\ldots$ & $\ldots$ & 6.78 & 6.78 & 5.56 & 90.0 & 90.0 & 120.0 \\
\hline 39 & $\mathrm{BaTiS}_{3}$ & P63/mmc(194) & $\mathrm{BaNiO} 3$ & Metal & $\cdots$ & $\cdots$ & $\cdots$ & $\cdots$ & $\ldots$ & $\cdots$ & $\ldots$ & 6.79 & 6.79 & 5.84 & 90.0 & 90.0 & 120.0 \\
\hline 40 & $\mathrm{CeScS}_{3}$ & Pna21(33) & Perovskite-ScYS3 & Metal & $\ldots$ & $\ldots$ & $\ldots$ & $\ldots$ & $\ldots$ & $\ldots$ & $\ldots$ & 7.22 & 6.53 & 9.64 & 90.0 & 90.0 & 90.0 \\
\hline 41 & $\mathrm{EuZrS}_{3}$ & Pnma(62) & $\mathrm{NH} 4 \mathrm{CdCl} 3 / \mathrm{Sn} 2 \mathrm{~S} 3$ & Metal & $\ldots$ & $\ldots$ & $\ldots$ & $\ldots$ & $\ldots$ & $\ldots$ & $\ldots$ & 6.74 & 7.13 & 9.79 & 90.0 & 90.0 & 90.0 \\
\hline 42 & $\mathrm{CeYbS}_{3}$ & Pnma(62) & CeErS3 & Metal & .. & $\ldots$ & $\ldots$ & $\ldots$ & $\ldots$ & $\ldots$ & $\ldots$ & 3.94 & 16.37 & 20.96 & 90.0 & 90.0 & 90.0 \\
\hline 43 & $\mathrm{EuZrSe}_{3}$ & $\operatorname{Pnma}(62)$ & $\mathrm{NH} 4 \mathrm{CdCl} 3 / \mathrm{Sn} 2 \mathrm{~S} 3$ & Metal & $\ldots$ & $\ldots$ & $\cdots$ & $\ldots$ & $\ldots$ & $\ldots$ & $\ldots$ & 3.97 & 8.91 & 14.47 & 90.0 & 90.0 & 90.0 \\
\hline 44 & $\mathrm{LaYbSe}_{3}$ & $\mathrm{Cmcm}(63)$ & ScUS3 & Metal & $\ldots$ & $\ldots$ & $\ldots$ & $\ldots$ & $\ldots$ & $\ldots$ & $\ldots$ & 4.20 & 14.56 & 9.30 & 90.0 & 90.0 & 90.0 \\
\hline 45 & $\mathrm{NdYbSe}_{3}$ & $\mathrm{Cmcm}(63)$ & ScUS3 & Metal & $\ldots$ & $\ldots$ & $\ldots$ & $\ldots$ & $\ldots$ & $\ldots$ & $\ldots$ & 4.12 & 14.49 & 9.29 & 90.0 & 90.0 & 90.0 \\
\hline 46 & $\mathrm{PrYbSe}_{3}$ & $\operatorname{Cmcm}(63)$ & ScUS3 & Metal & $\ldots$ & $\ldots$ & $\ldots$ & $\ldots$ & $\ldots$ & $\ldots$ & $\ldots$ & 4.15 & 14.53 & 9.30 & 90.0 & 90.0 & 90.0 \\
\hline 47 & $\mathrm{CeCrSe}_{3}$ & $\operatorname{Pnam}(62)$ & $\mathrm{NH} 4 \mathrm{CdCl} 3 / \mathrm{Sn} 2 \mathrm{~S} 3$ & Metal & $\ldots$ & $\ldots$ & $\ldots$ & $\ldots$ & $\ldots$ & $\ldots$ & $\ldots$ & 3.98 & 8.15 & 13.65 & 90.0 & 90.0 & 90.0 \\
\hline 48 & $\mathrm{CeYbSe}_{3}$ & $\operatorname{Cmcm}(63)$ & ScUS3 & Metal & ... & $\ldots$ & $\ldots$ & $\ldots$ & $\ldots$ & $\ldots$ & $\ldots$ & 4.04 & 13.87 & 9.66 & 90.0 & 90.0 & 90.0 \\
\hline 49 & $\mathrm{GaCrSe}_{3}$ & Pnma(62) & CuTaS3 & Metal & $\cdots$ & $\cdots$ & $\ldots$ & $\ldots$ & $\ldots$ & $\ldots$ & $\ldots$ & 3.80 & 10.39 & 12.57 & 90.0 & 90.0 & 90.0 \\
\hline 50 & $\mathrm{SmYbSe}_{3}$ & $\mathrm{Cmcm}(63)$ & ScUS3 & Metal & $\ldots$ & $\ldots$ & $\ldots$ & $\ldots$ & $\ldots$ & $\ldots$ & $\ldots$ & 4.07 & 14.44 & 9.26 & 90.0 & 90.0 & 90.0 \\
\hline 51 & $\mathrm{NdLuSe}_{3}$ & $\mathrm{Cmcm}(63)$ & ScUS3 & Metal & ... & $\ldots$ & $\ldots$ & $\ldots$ & $\ldots$ & $\ldots$ & $\ldots$ & 4.03 & 13.13 & 9.97 & 90.0 & 90.0 & 90.0 \\
\hline 52 & $\mathrm{PrLuSe}_{3}$ & $\mathrm{Cmcm}(63)$ & ScUS3 & Metal & .. & $\ldots$ & $\ldots$ & $\ldots$ & $\ldots$ & $\ldots$ & $\ldots$ & 4.01 & 13.00 & 9.87 & 90.0 & 90.0 & 90.0 \\
\hline 53 & $\mathrm{BaTaSe}_{3}$ & $\mathrm{P} 63 / \mathrm{mmc}(194)$ & $\mathrm{BaNiO} 3$ & Metal & $\ldots$ & $\ldots$ & $\ldots$ & $\ldots$ & $\ldots$ & $\cdots$ & & 7.13 & 7.13 & 5.99 & 90.0 & 90.0 & 120.0 \\
\hline 54 & $\mathrm{BaTiSe}_{3}$ & P63/mmc(194) & $\mathrm{BaNiO} 3$ & Metal & $\ldots$ & $\ldots$ & $\ldots$ & $\ldots$ & & $\cdots$ & $\cdots$ & 7.05 & 7.05 & 6.03 & 90.0 & 90.0 & 120.0 \\
\hline 55 & $\mathrm{LaCrSe}_{3}$ & Pnma(62) & $\mathrm{NH} 4 \mathrm{CdCl} 3 / \mathrm{Sn} 2 \mathrm{~S} 3$ & Metal & . & $\ldots$ & $\ldots$ & $\ldots$ & $\ldots$ & $\ldots$ & $\ldots$ & 8.22 & 4.01 & 13.79 & 90.0 & 90.0 & 90.0 \\
\hline
\end{tabular}


Table S2: Calculated PF's for $\mathrm{GdFeO}_{3}$ and $\mathrm{Sn}_{2} \mathrm{~S}_{3}$ prototypes

\begin{tabular}{|c|c|c|c|c|c|c|c|c|}
\hline No. & Material & Prototype & Pfxx(hole) & Pfyy(hole) & Pfzz(hole) & $\operatorname{Pfxx}(\mathrm{elec})$ & Pfyy(elec) & Pfzz(elec) \\
\hline 1 & $\mathrm{PbHfS}_{3}$ & $\mathrm{GdFeO}_{3}$ & 1.45 & 1.2 & 0.88 & 0.21 & 2.31 & 0.27 \\
\hline 2 & $\mathrm{PbHfS}_{3}$ & $\mathrm{Sn}_{2} \mathrm{~S}_{3}$ & 1.1 & 1.8 & 0.2 & 1.5 & 5.0 & 0.1 \\
\hline 3 & $\mathrm{PbZrS}_{3}$ & $\mathrm{GdFeO}_{3}$ & 1.35 & 1.11 & 0.86 & 1.14 & 2.46 & 2.24 \\
\hline 4 & $\mathrm{PbZrS}_{3}$ & $\mathrm{Sn}_{2} \mathrm{~S}_{3}$ & 0.79 & 2.03 & 0.18 & 0.14 & 3.5 & 0.08 \\
\hline 5 & $\mathrm{SnHfS}_{3}$ & $\mathrm{GdFeO}_{3}$ & 0.87 & 1.19 & 2.83 & 0.21 & 0.16 & 0.29 \\
\hline 6 & $\mathrm{SnHfS}_{3}$ & $\mathrm{Sn}_{2} \mathrm{~S}_{3}$ & 0.87 & 0.85 & 0.28 & 5.18 & 0.03 & 0.05 \\
\hline 7 & $\mathrm{SnZrS}_{3}$ & $\mathrm{GdFeO}_{3}$ & 1.07 & 1.28 & 2.4 & 0.23 & 2.69 & 0.26 \\
\hline 8 & $\mathrm{SnZrS}_{3}$ & $\mathrm{Sn}_{2} \mathrm{~S}_{3}$ & 1.0 & 0.85 & 0.41 & 4.29 & 0.07 & 0.1 \\
\hline 9 & $\mathrm{BaHfS}_{3}$ & $\mathrm{GdFeO}_{3}$ & 2.5 & 2.44 & 2.97 & 2.05 & 2.32 & 2.18 \\
\hline 10 & $\mathrm{BaHfS}_{3}$ & $\mathrm{Sn}_{2} \mathrm{~S}_{3}$ & 0.89 & 0.49 & 0.09 & 0.24 & 0.76 & 0.17 \\
\hline 11 & $\mathrm{BaZrS}_{3}$ & $\mathrm{GdFeO}_{3}$ & 2.59 & 2.45 & 3.0 & 2.02 & 2.37 & 2.59 \\
\hline 12 & $\mathrm{BaZrS}_{3}$ & $\mathrm{Sn}_{2} \mathrm{~S}_{3}$ & 0.89 & 0.52 & 0.08 & 0.27 & 0.72 & 0.18 \\
\hline 13 & $\mathrm{SrHfS}_{3}$ & $\mathrm{GdFeO}_{3}$ & 1.02 & 1.24 & 0.74 & 1.72 & 2.19 & 2.88 \\
\hline 14 & $\mathrm{SrHfS}_{3}$ & $\mathrm{Sn}_{2} \mathrm{~S}_{3}$ & 0.71 & 0.41 & 0.09 & 0.27 & 0.79 & 0.14 \\
\hline 15 & $\mathrm{SrZrS}_{3}$ & $\mathrm{GdFeO}_{3}$ & 0.86 & 0.75 & 0.63 & 1.46 & 1.97 & 2.53 \\
\hline 16 & $\mathrm{SrZrS}_{3}$ & $\mathrm{Sn}_{2} \mathrm{~S}_{3}$ & 0.78 & 0.44 & 0.08 & 0.29 & 0.7 & 0.15 \\
\hline
\end{tabular}


Table S3: Bader charge (shown with a bold font) and direction-dependent Born effective charge $\left(Z_{x x}^{*} / Z_{y y}^{*} / Z_{z z}^{*}\right)$ for $A B X_{3}$ compounds. By symmetry, the prototypes $\mathrm{GdFeO}_{3}$ and $\mathrm{Sn}_{2} \mathrm{~S}_{3}$ have two and three different $X$ types, respectively

\begin{tabular}{lccccc}
\hline Compound & $\mathrm{A}$ & $\mathrm{B}$ & $\mathrm{X} 1$ & $\mathrm{X} 2$ & $\mathrm{X} 3$ \\
\hline $\mathrm{BaHfS}_{3}\left(\mathrm{GdFeO}_{3}\right)$ & $\mathbf{0 . 6}$ & $\mathbf{1 . 1 4}$ & $\mathbf{- 1 . 1}$ & $\mathbf{- 0 . 1 5}$ & - \\
& $2.7 / 2.7 / 2.7$ & $7.5 / 7.5 / 7.5$ & $-2.1 /-2.1 /-6.1$ & $-4.1 /-4.1 /-2.1$ & - \\
$\mathrm{BaZrS}_{3}\left(\mathrm{GdFeO}_{3}\right)$ & $\mathbf{1 . 2}$ & $\mathbf{1 . 7}$ & $\mathbf{- 1 . 1}$ & $\mathbf{- 0 . 9}$ & - \\
& $2.7 / 2.7 / 2$. & $7.9 / 8.1 / 8.1$ & $-2.2 /-2.0 /-6.5$ & $-4.2 /-4.4 /-2.2$ & - \\
$\mathrm{SrZrO}_{3}\left(\mathrm{GdFeO}_{3}\right)$ & $\mathbf{1 . 5}$ & $\mathbf{2 . 7}$ & $\mathbf{- 1 . 4}$ & $\mathbf{- 1 . 5}$ & - \\
& $2.5 / 2.5 / 2.4$ & $6.0 / 5.9 / 6$. & $-3.3 /-3.3 /-1.9$ & $-2.0 /-1.8 /-4.2$ & - \\
$\left.\mathrm{SrZrS}_{3}\left(\mathrm{GdFeO}_{3}\right)\right)$ & $2.5 / 2.5 / 2.5$ & $7.1 / 7.8 / 7.6$ & $-3.7 /-4.3 /-2.0$ & $-2.1 /-1.8 /-6.1$ & - \\
& $\mathbf{0 . 7}$ & 1.6 & & & - \\
$\mathrm{SbGaS}_{3}\left(\mathrm{Sn}_{2} \mathrm{~S}_{3}\right)$ & $\mathbf{1 . 2}$ & $\mathbf{1 . 3}$ & $\mathbf{- 0 . 8}$ & $\mathbf{- 0 . 8}$ & $\mathbf{- 0 . 8}$ \\
& $6.2 / 2.8 / 2.3$ & $2.7 / 2.4 / 2.1$ & $-2.7 /-1.1 /-1.7$ & $-3.6 /-1.5 /-1.0$ & $-2.7 /-2.7 /-1.7$ \\
$\mathrm{SrZrS}_{3}\left(\mathrm{Sn}_{2} \mathrm{~S}_{3}\right)$ & $\mathbf{1 . 4}$ & $\mathbf{0 . 9}$ & $\mathbf{- 1 . 2}$ & $\mathbf{- 1 . 1}$ & $\mathbf{- 1 . 0}$ \\
$\mathrm{TaTlSe}_{3}\left(\mathrm{Sn}_{2} \mathrm{~S}_{3}\right)$ & $\mathbf{1 . 9}$ & $\mathbf{0 . 5}$ & $\mathbf{- 0 . 8}$ & $\mathbf{- 0 . 9}$ & $\mathbf{- 0 . 6}$ \\
\hline
\end{tabular}

Table S4: Power Factor calculation with/without spin-orbit coupling (Bold Font indicates SOC)

\begin{tabular}{llccccc}
\hline Material & PF-hole(xx) & PF-hole(yy) & PF-hole(zz) & PF-elec(xx) & PF-elec(yy) & PF-elec(zz) \\
\hline BaZrS3 & 2.59 & 2.45 & 2.96 & 2.07 & 2.37 & 2.59 \\
& $\mathbf{1 . 9 6}$ & $\mathbf{1 . 7 8}$ & $\mathbf{2 . 3 4}$ & $\mathbf{1 . 8 5}$ & $\mathbf{2 . 2 8}$ & $\mathbf{2 . 4 2}$ \\
PbHfS3 & 0.91 & 1.75 & 0.16 & 0.19 & 4.23 & 0.04 \\
& $\mathbf{1 . 0 5}$ & $\mathbf{1 . 7 9}$ & $\mathbf{0 . 1 8}$ & $\mathbf{1 . 4 9}$ & $\mathbf{4 . 9 8}$ & $\mathbf{0 . 0 7}$ \\
SbGaS3 & 4.73 & 0.38 & 0.17 & 2.35 & 0.31 & 0.00 \\
& $\mathbf{4 . 7 8}$ & $\mathbf{0 . 4 0}$ & $\mathbf{0 . 1 8}$ & $\mathbf{2 . 2 3}$ & $\mathbf{0 . 2 7}$ & $\mathbf{0 . 3 7}$ \\
SnZrS3 & 1.00 & 0.80 & 0.26 & 4.39 & 0.07 & 0.06 \\
& $\mathbf{0 . 9 7}$ & $\mathbf{0 . 8 5}$ & $\mathbf{0 . 4 1}$ & $\mathbf{4 . 2 9}$ & $\mathbf{0 . 0 7}$ & $\mathbf{0 . 1 0}$ \\
SrTeS3 & 2.83 & 1.43 & 2.97 & 3.30 & 0.24 & 0.42 \\
& $\mathbf{2 . 8 7}$ & $\mathbf{1 . 3 6}$ & $\mathbf{2 . 8 5}$ & $\mathbf{3 . 1 2}$ & $\mathbf{0 . 1 7}$ & $\mathbf{0 . 3 9}$ \\
TaNaS3 & 3.44 & 3.98 & 2.47 & 2.17 & 3.48 & 1.44 \\
& $\mathbf{2 . 1 3}$ & $\mathbf{2 . 5 6}$ & $\mathbf{1 . 9 9}$ & $\mathbf{2 . 1 1}$ & $\mathbf{3 . 3 8}$ & $\mathbf{1 . 4 9}$ \\
\hline
\end{tabular}



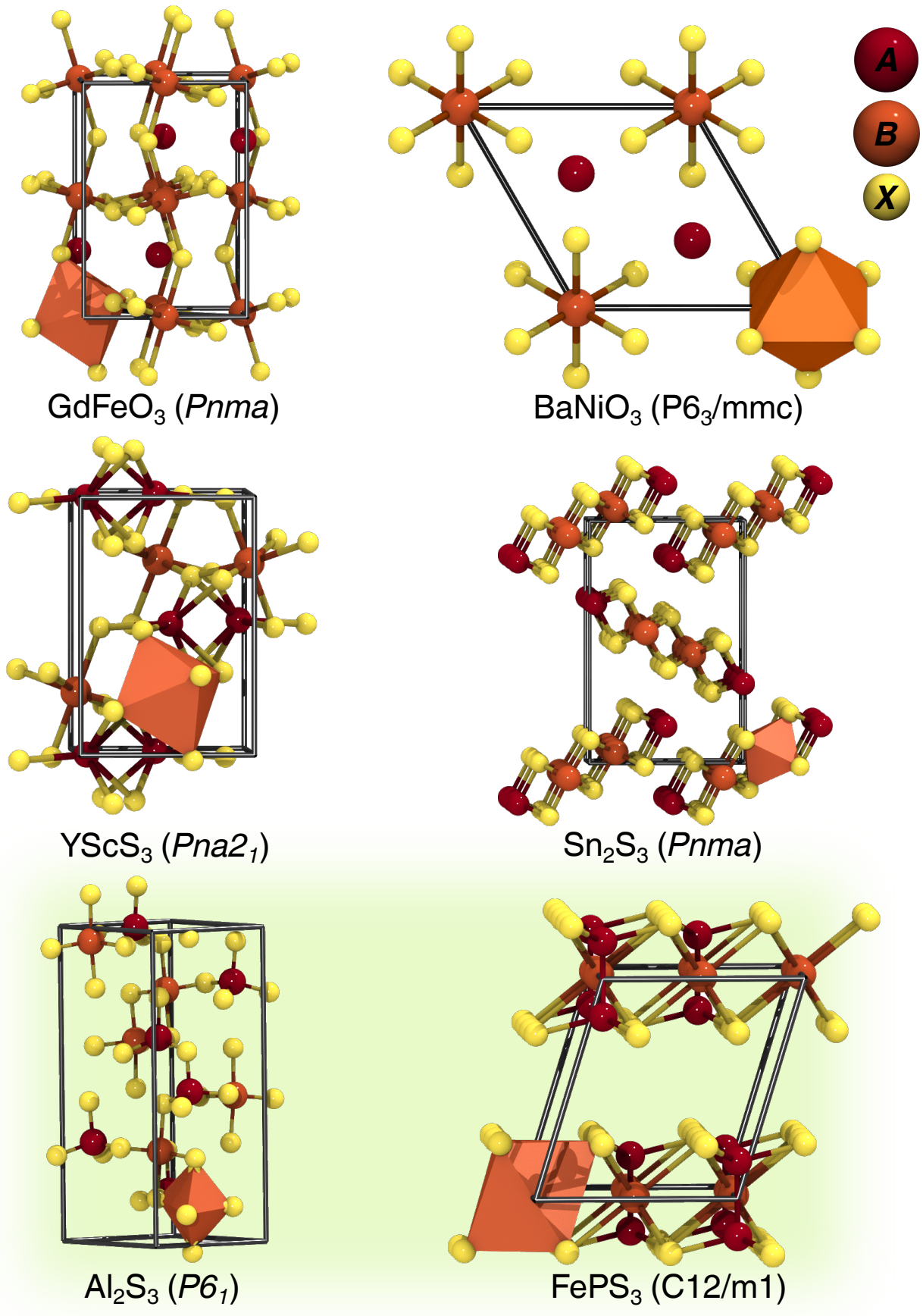


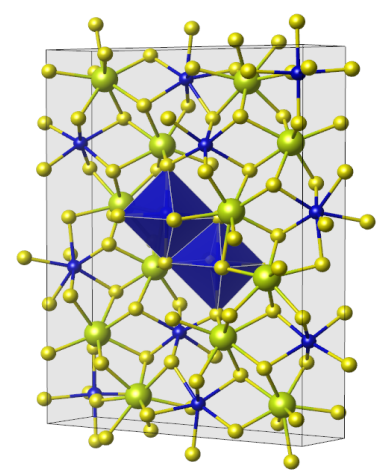

$\mathrm{CeErS}_{3}$

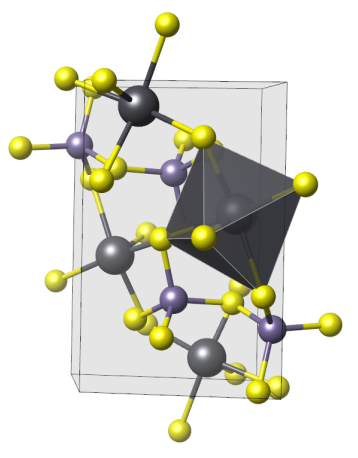

$\mathrm{GePbS}_{3}$
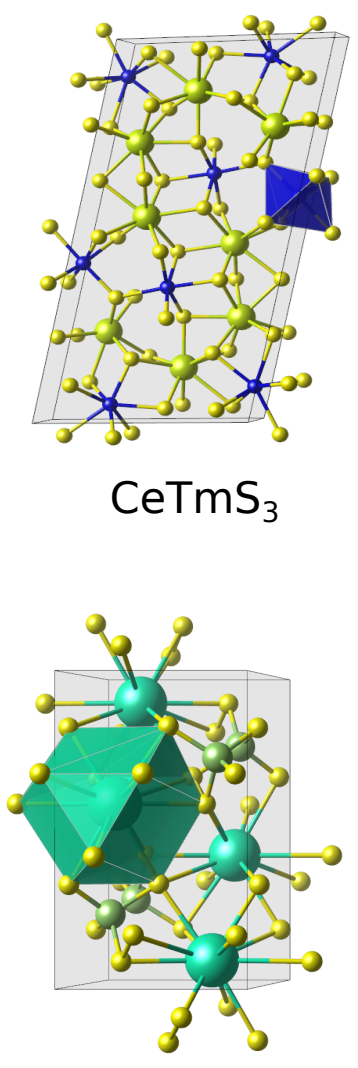

$\mathrm{RbBSe}_{3}$
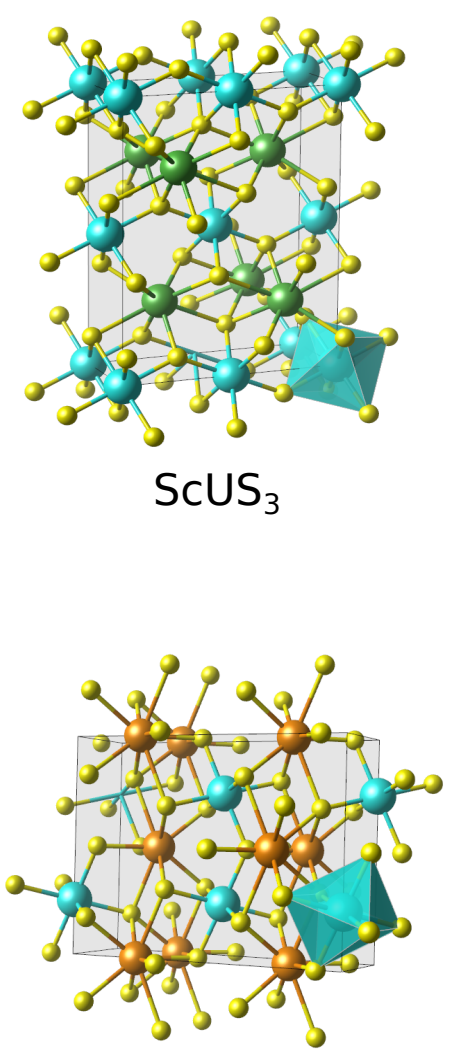

$\mathrm{NdYbS}_{3}$

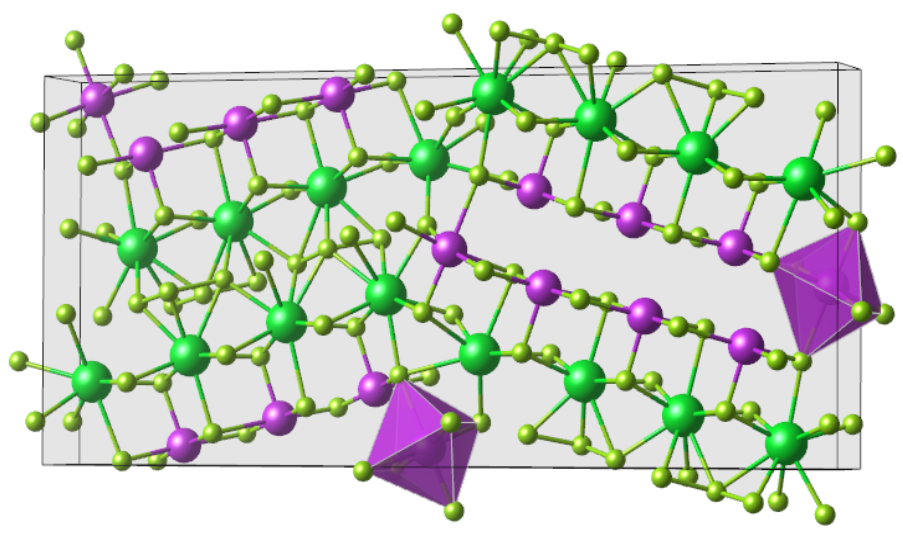

$\mathrm{SrBiSe}_{3}$ 


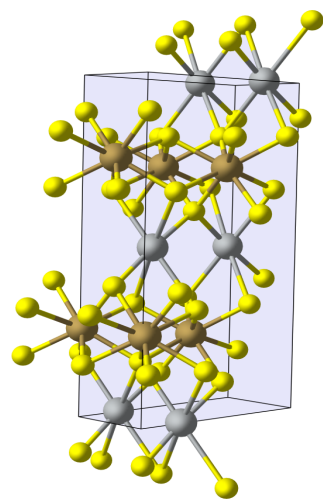

Pyroxene-CalrO 3

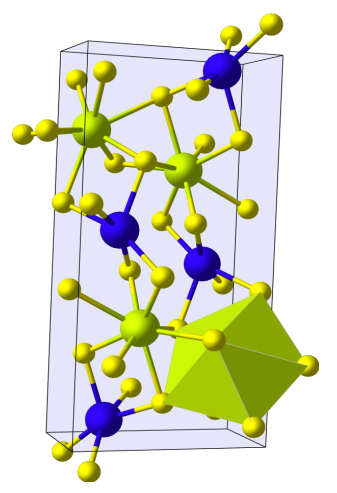

$\mathrm{La}_{2} \mathrm{~S}_{3}$

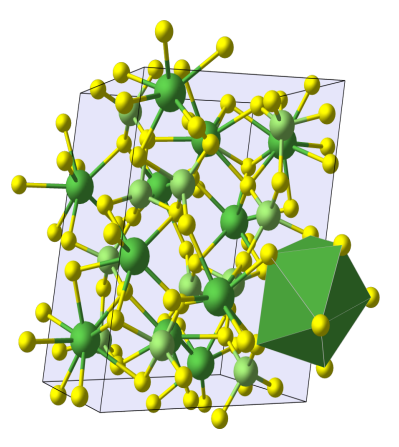

$\mathrm{LaGaS}_{3}$

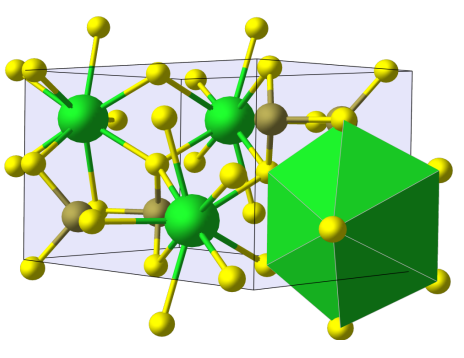

Ragonite- $\mathrm{CaCO}_{3}$

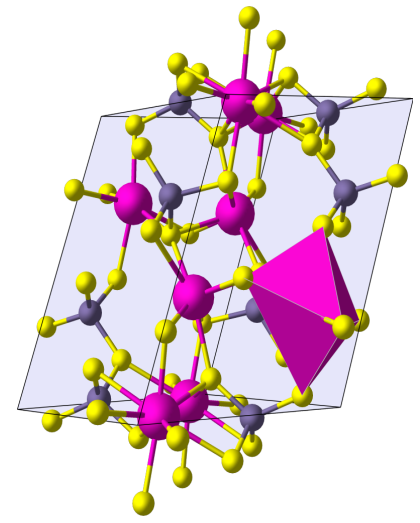

$\mathrm{CaSiO}_{3}$

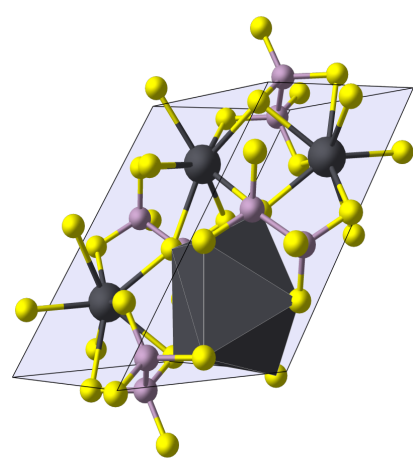

$\mathrm{PbPS}_{3}$

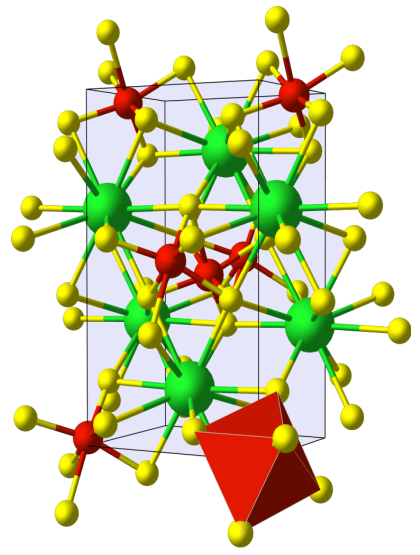

$\mathrm{BaVS}_{3}$

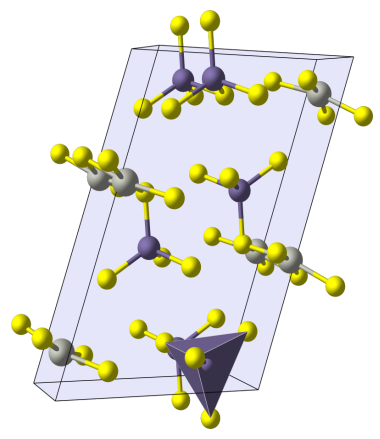

$\mathrm{Dy}_{3} \mathrm{Ni}_{2}$

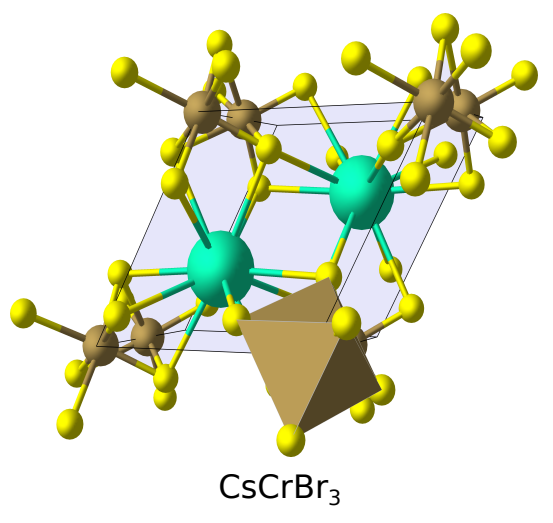

Figure S 1: The prototypes of the studied compounds. The first four ones are known as perovskite structure type. 


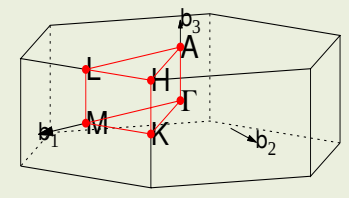

$\mathrm{Al}_{2} \mathrm{~S}_{3}$

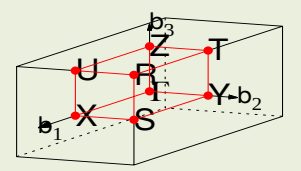

$\mathrm{CuTaS}_{3}$
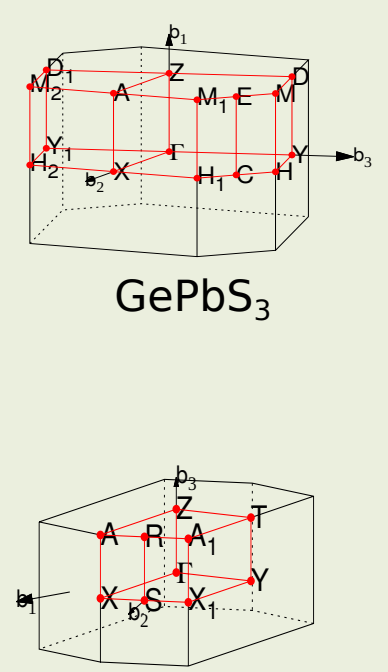

$\mathrm{ScUS}_{3}$

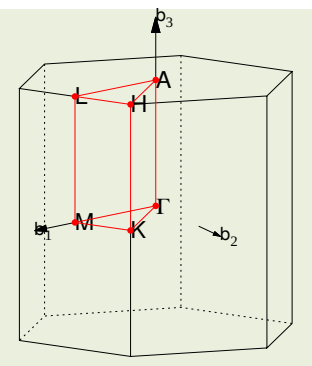

$\mathrm{BaNiO}_{3}$

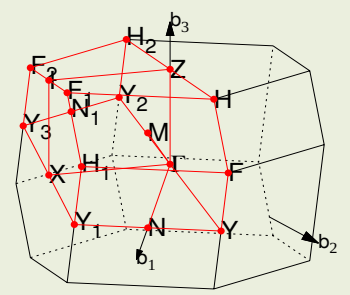

$\mathrm{FePS}_{3}$

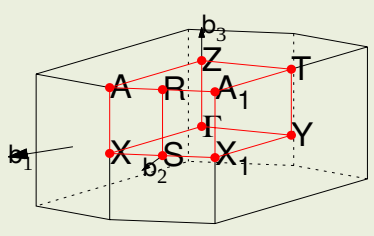

$\mathrm{NdYbS}_{3}$

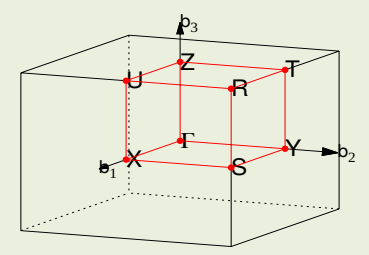

$\mathrm{YScS}_{3}$

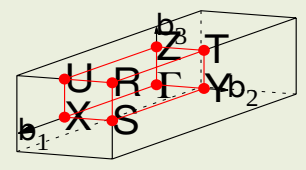

$\mathrm{CeErS}_{3}$

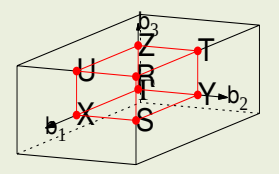

$\mathrm{GdFeO}_{3}$

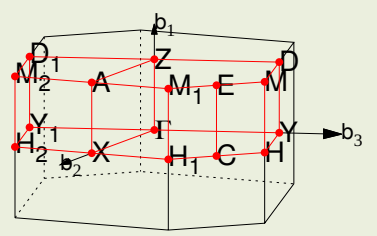

$\mathrm{RbBSe}_{3}$

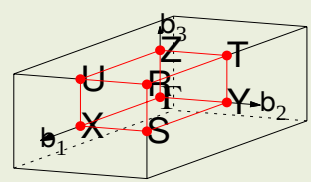

$\mathrm{Sn}_{3} \mathrm{~S}_{3}$

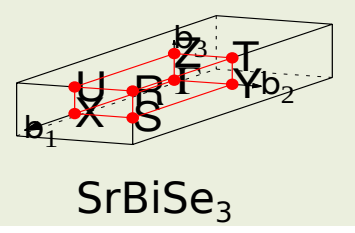

Figure S2: BZ direction all of prototypes 

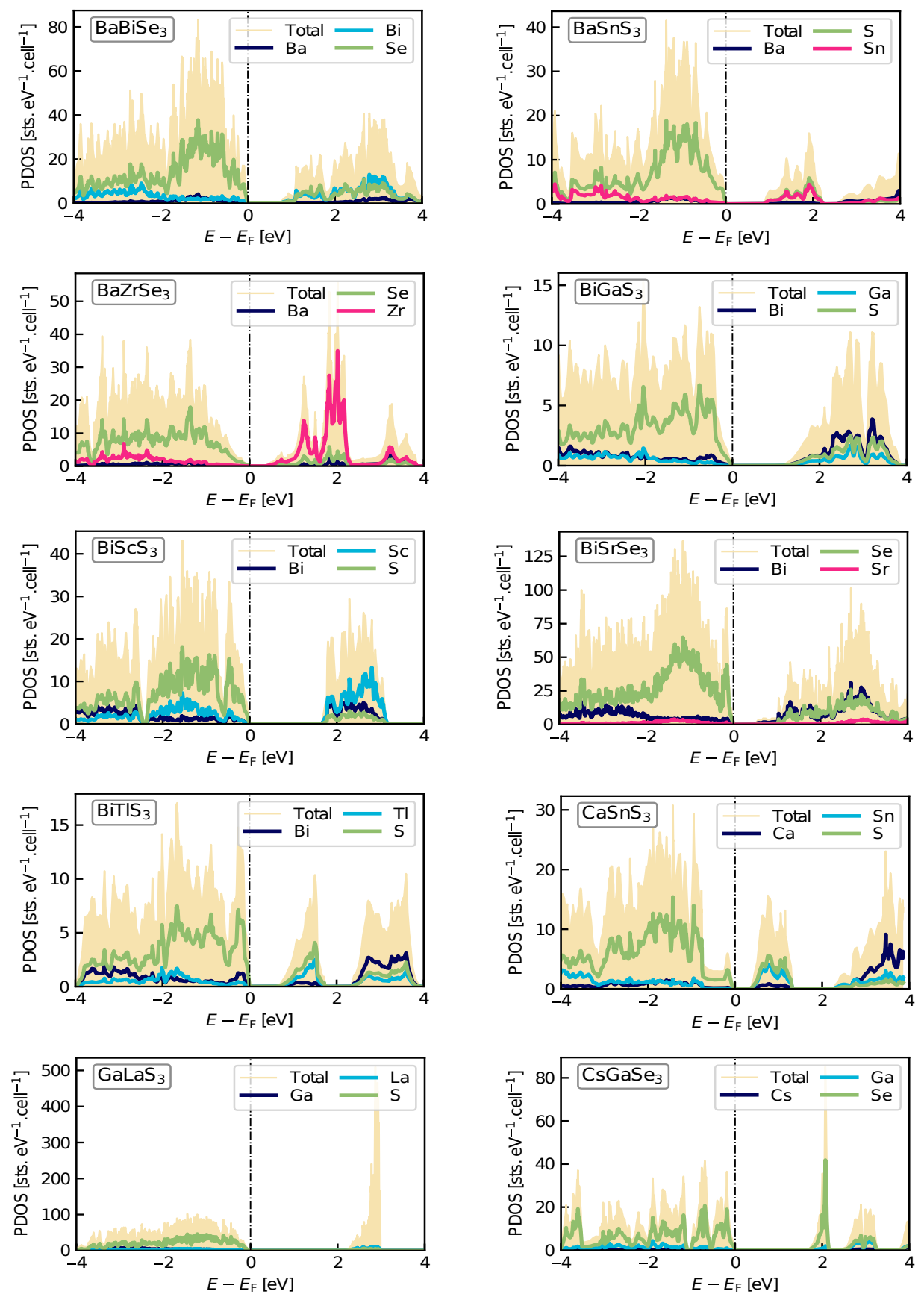

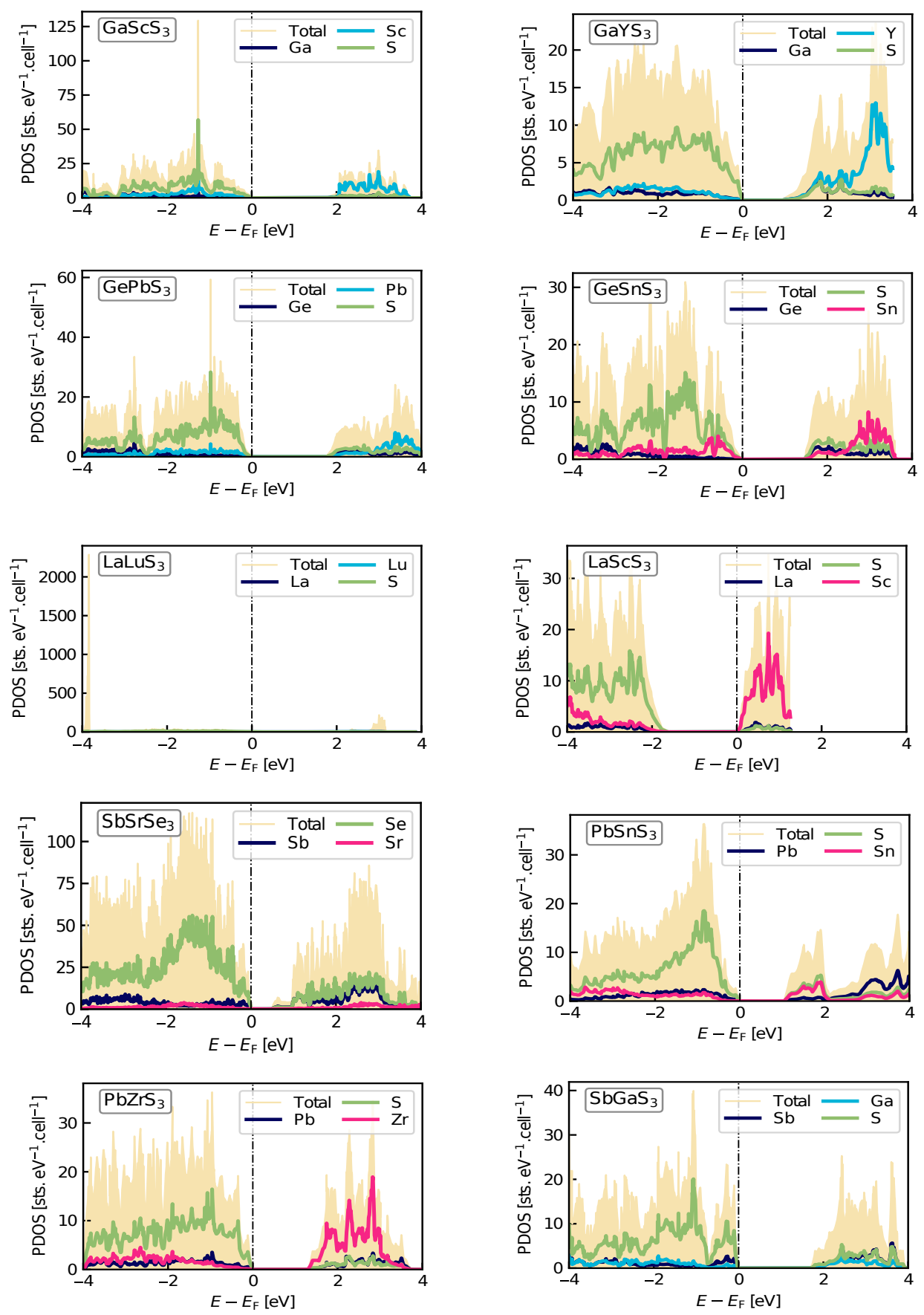

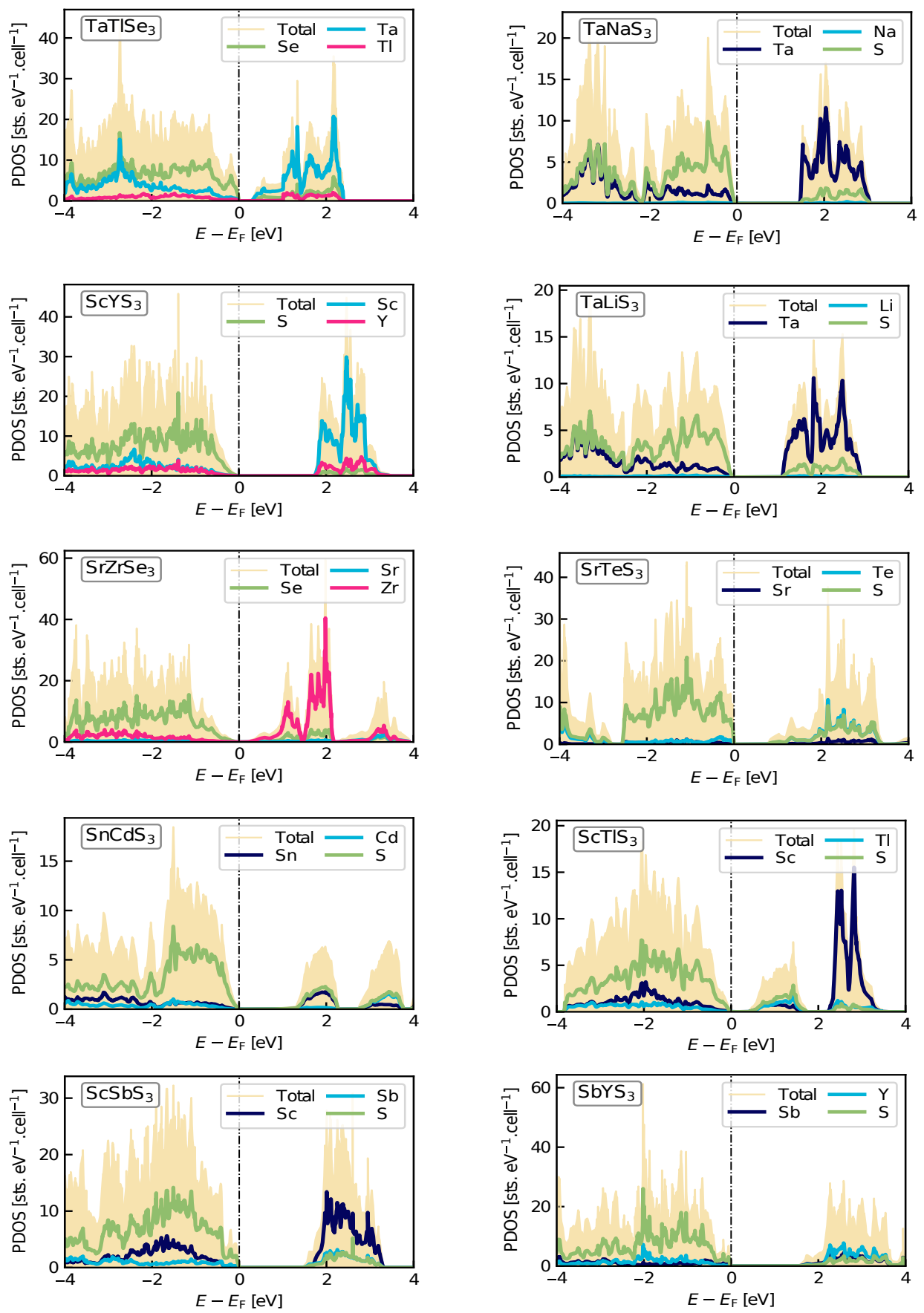

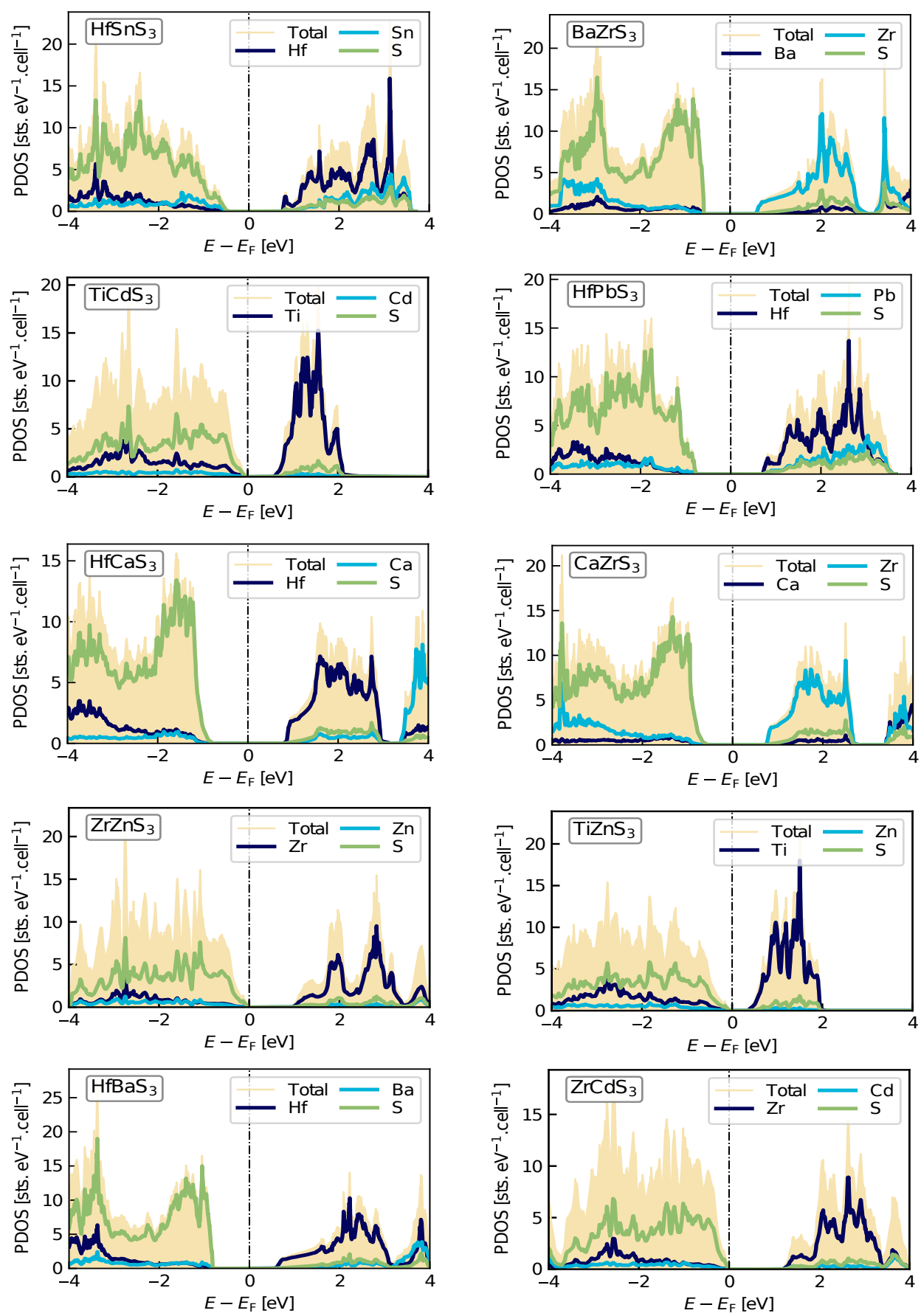

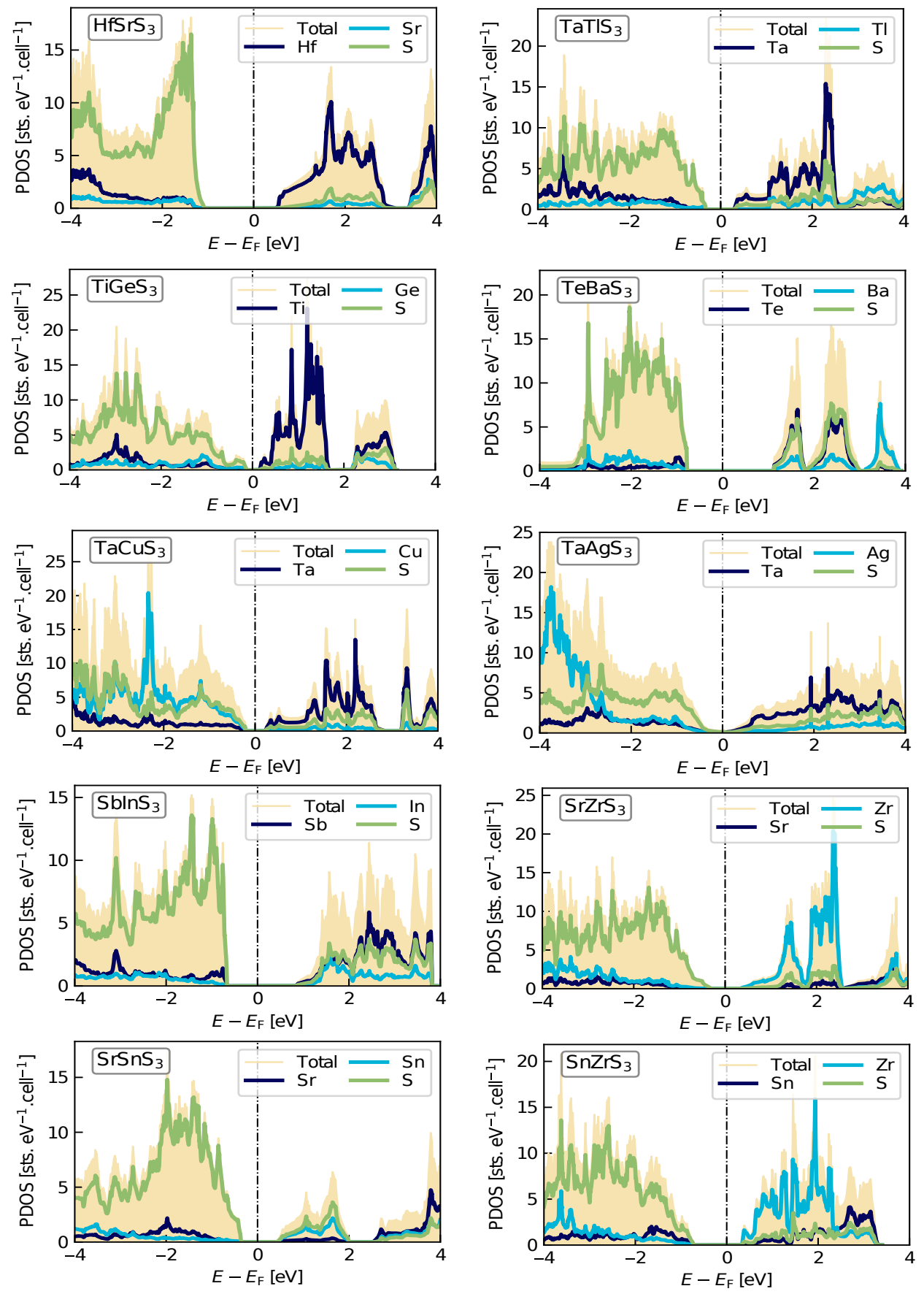

Figure S 3: Atom projected density of states (PDOS) for semiconducting chalcogenide perovskites. 

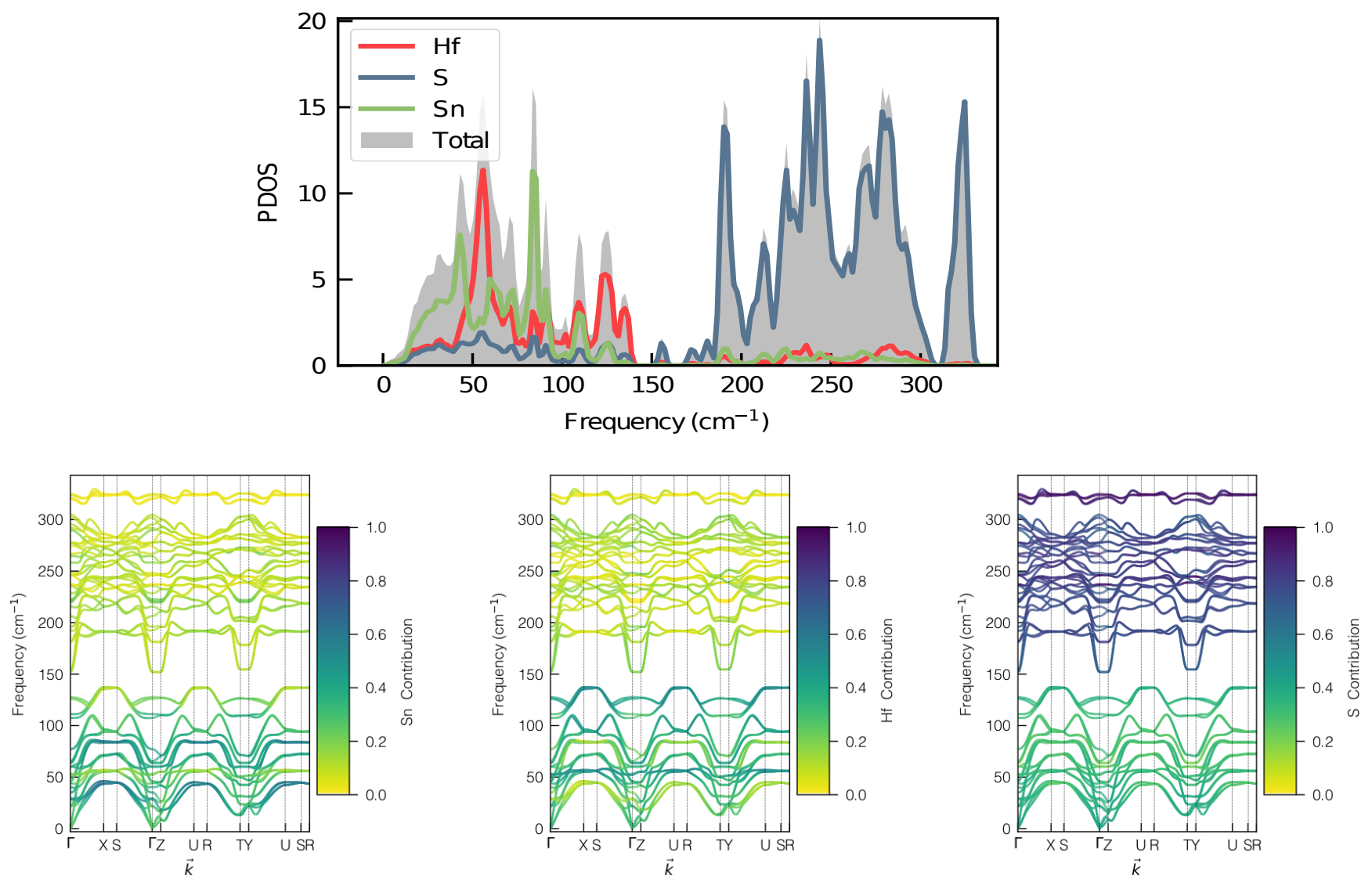

Figure S4: Calculated phonon dispersion of $\mathrm{SnHfS}_{3}$. 

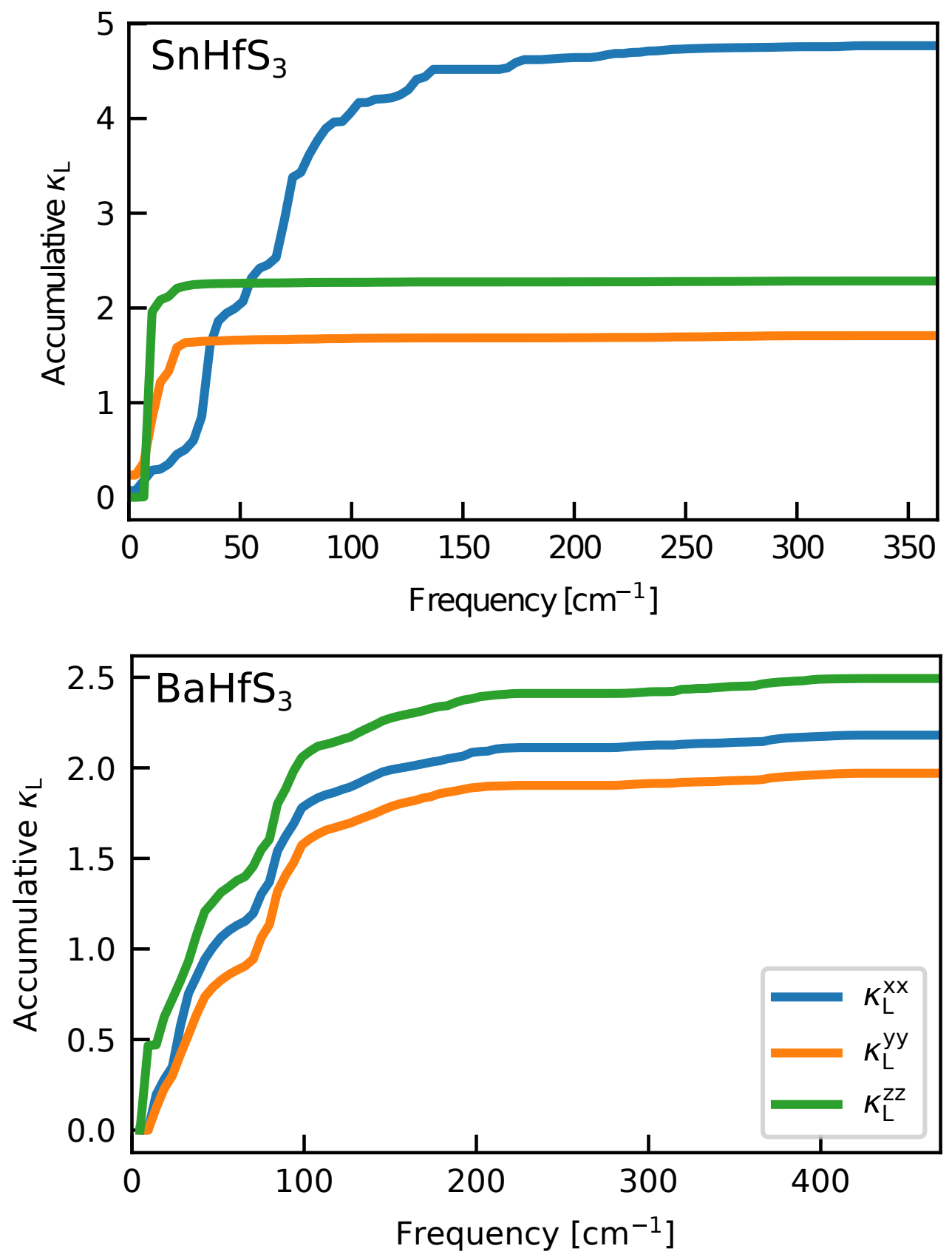

Figure S5: Calculated accumulative lattice thermal conductivity versus frequency. 

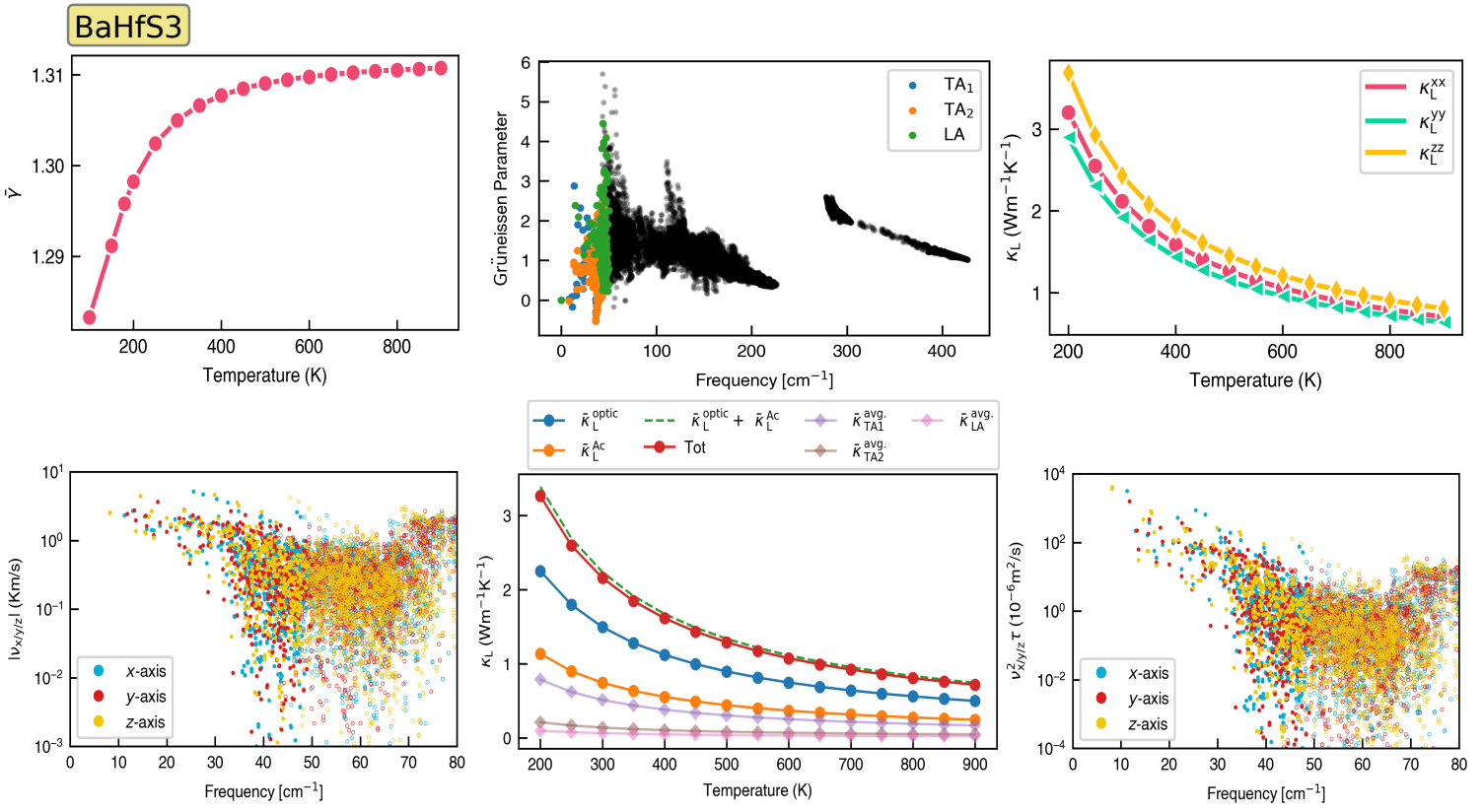

\section{HfSnS3}
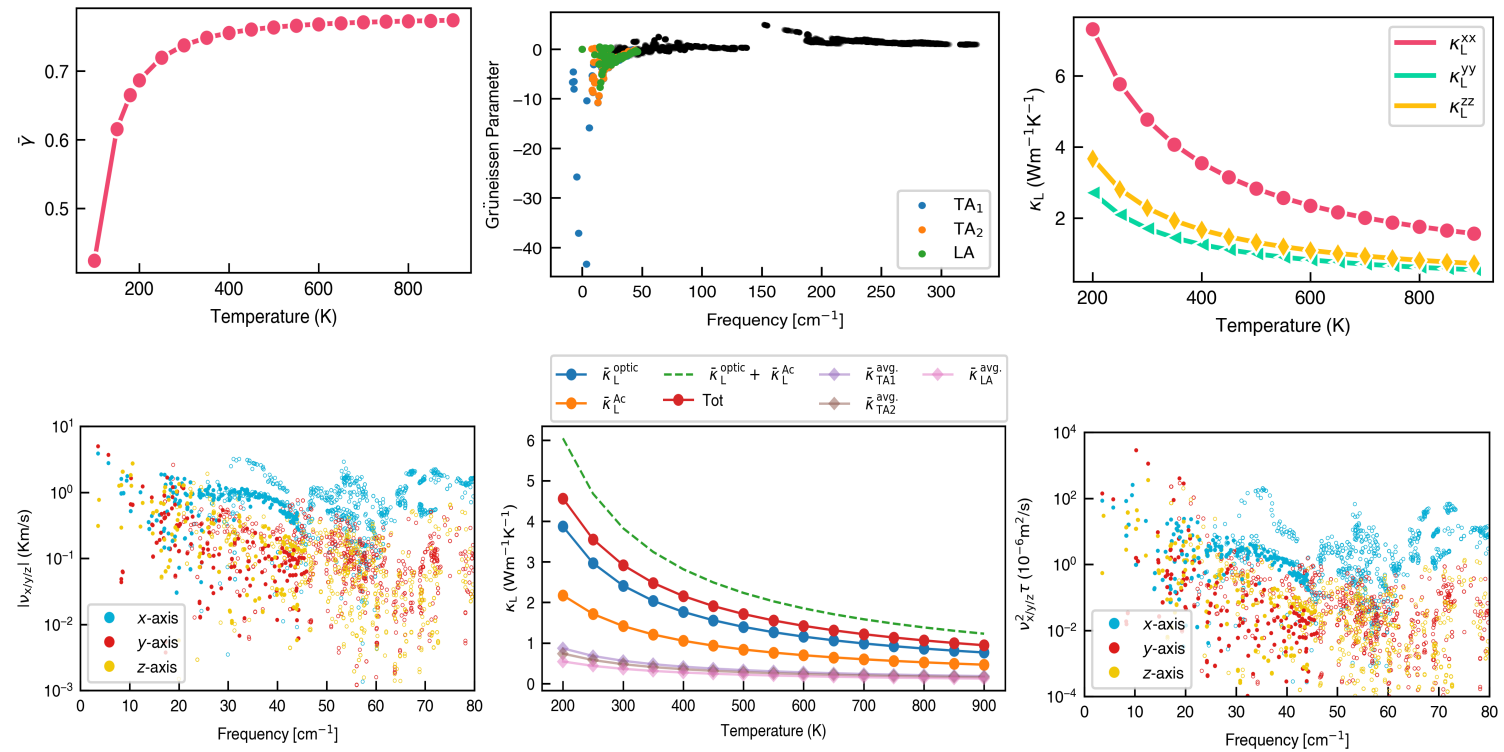

$\rightarrow \bar{\kappa}_{L}^{\text {optic }}---\bar{\kappa}_{L}^{\text {optic }}+\bar{K}_{L}^{A C} \multimap \bar{K}_{T A 1}^{\text {avg. }} \dashv \bar{K}_{L A}^{\text {avg. }}$
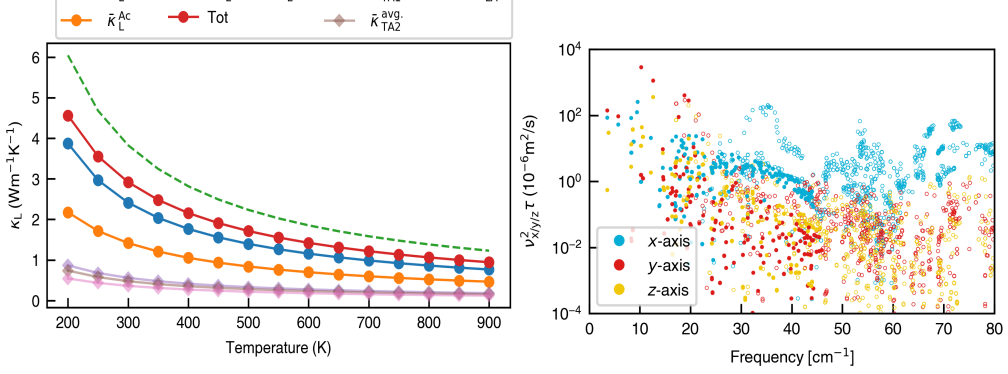

Figure S6: Thermal Proprieties cålculated for $\mathrm{BaHfS}_{3}$ and $\mathrm{SnHfS}_{3}$ 

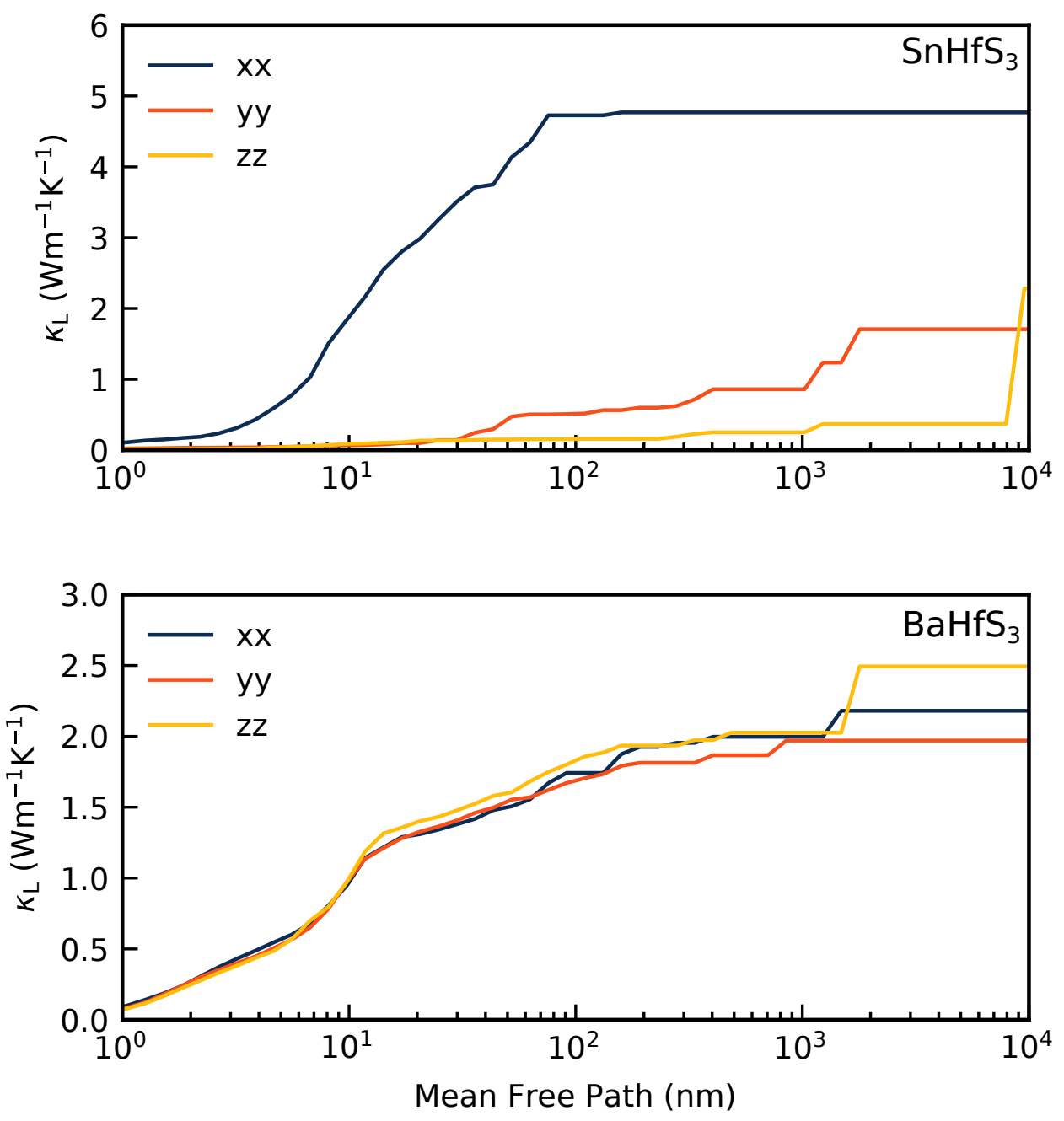

Figure S7: Calculated accumulative lattice thermal conductivity versus mean free path. 

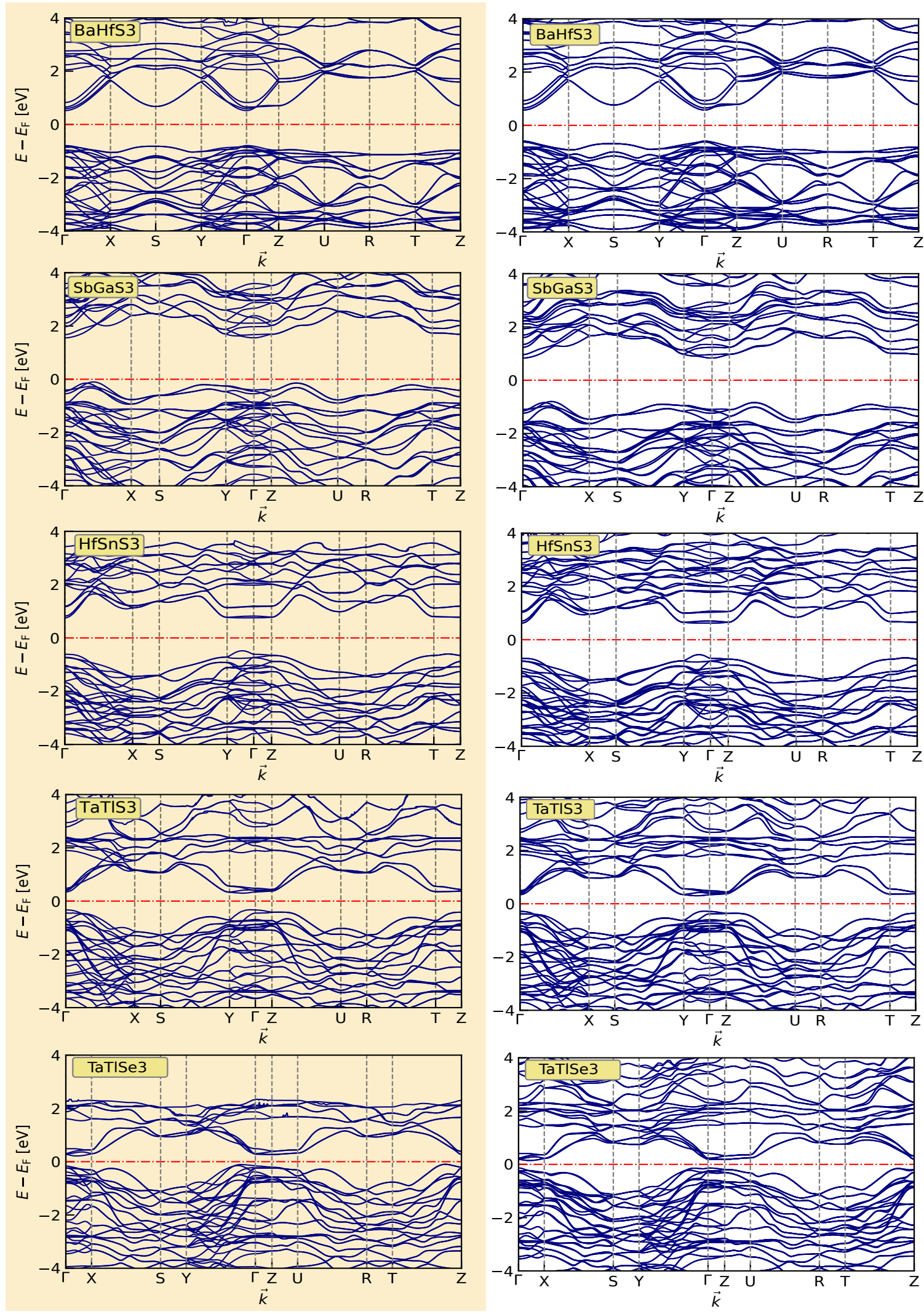

20

Figure S 8: Calculated band-structures with spin-orbit coupling and without SOC (Colored background). 

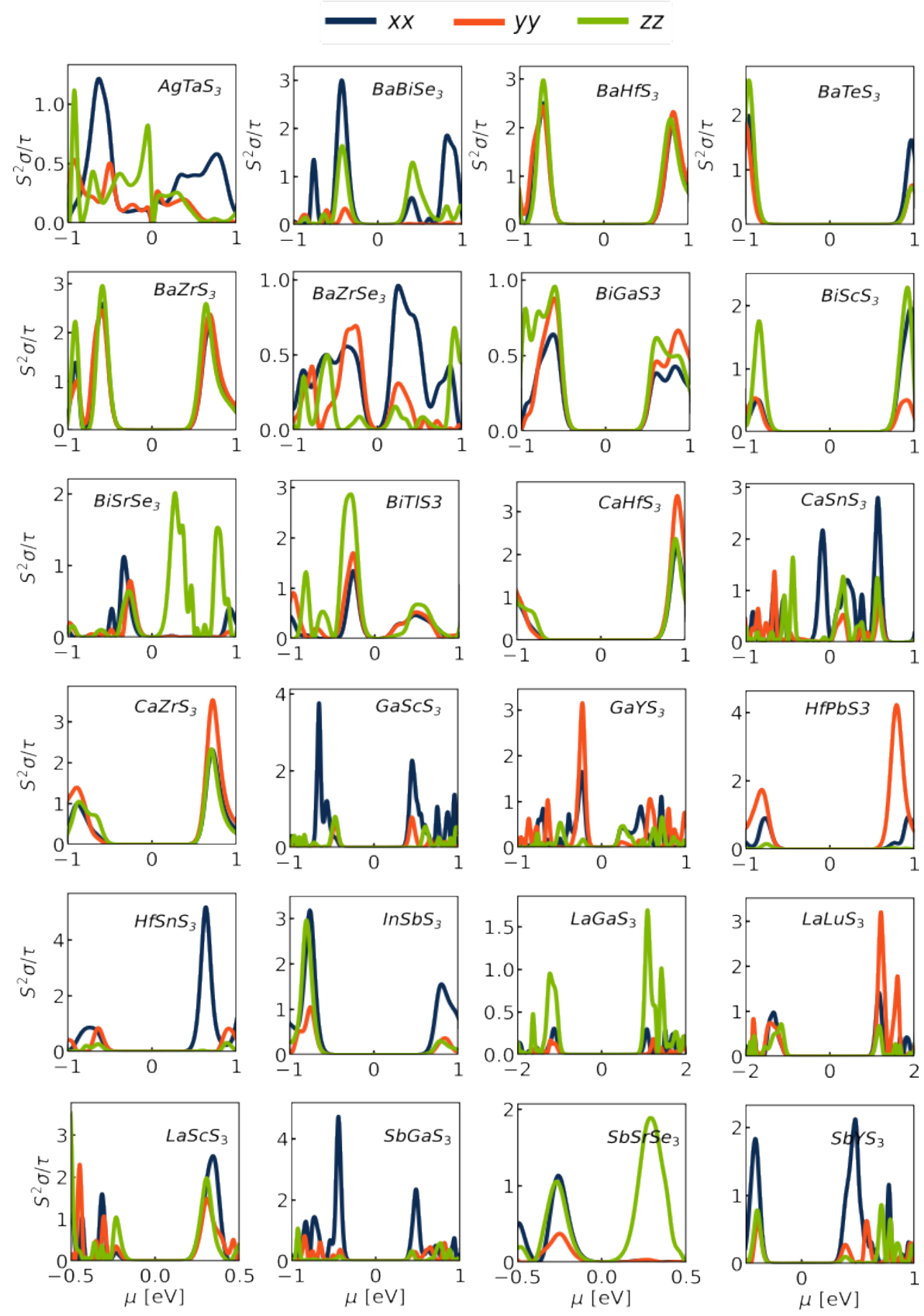

Continue on the Next Page 

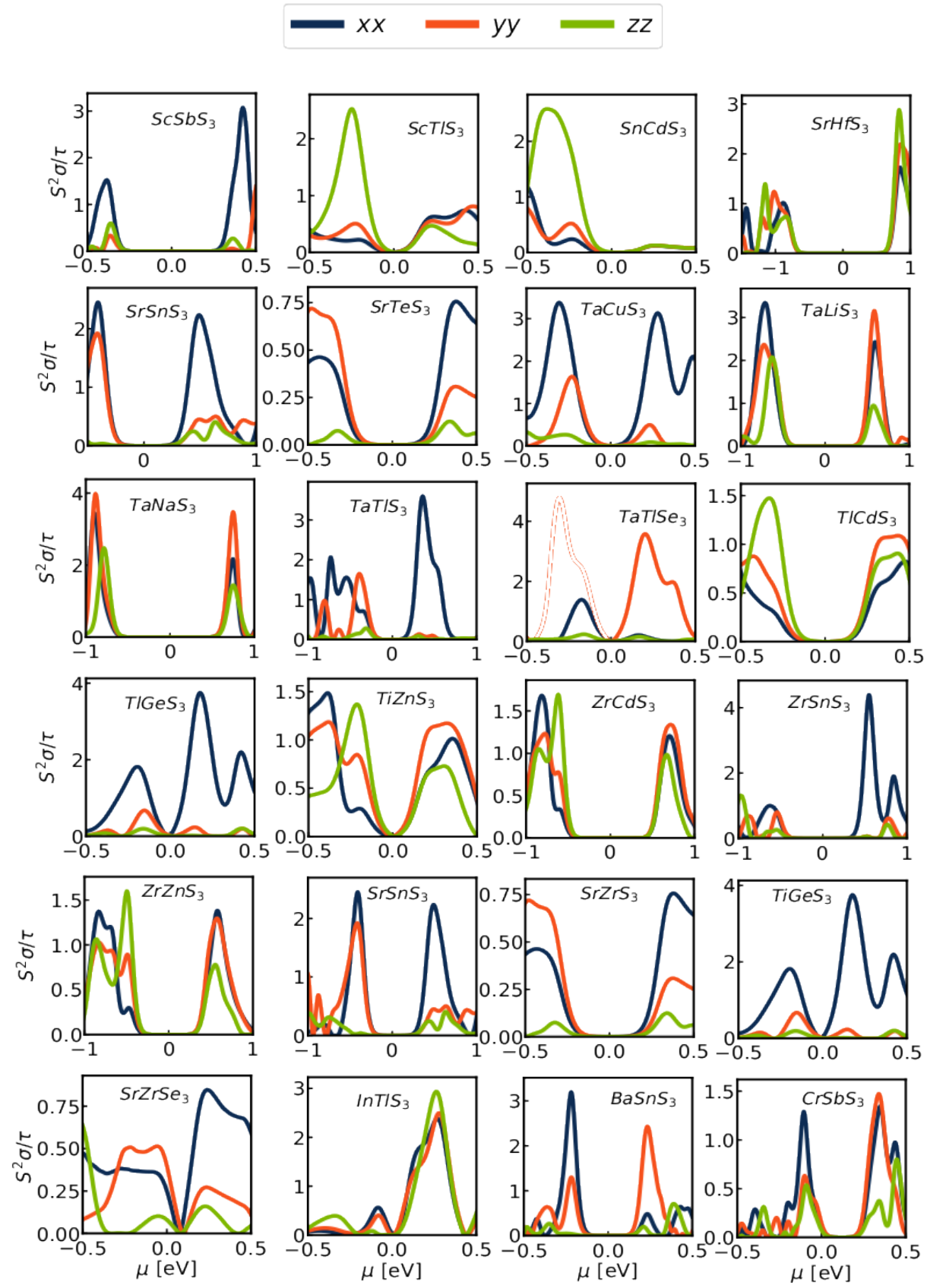

Figure S 9: Calculated transport coefficients of the studied materials assuming the constant relaxation time of $\tau=1 \times 10^{-14} \mathrm{~s}$. 

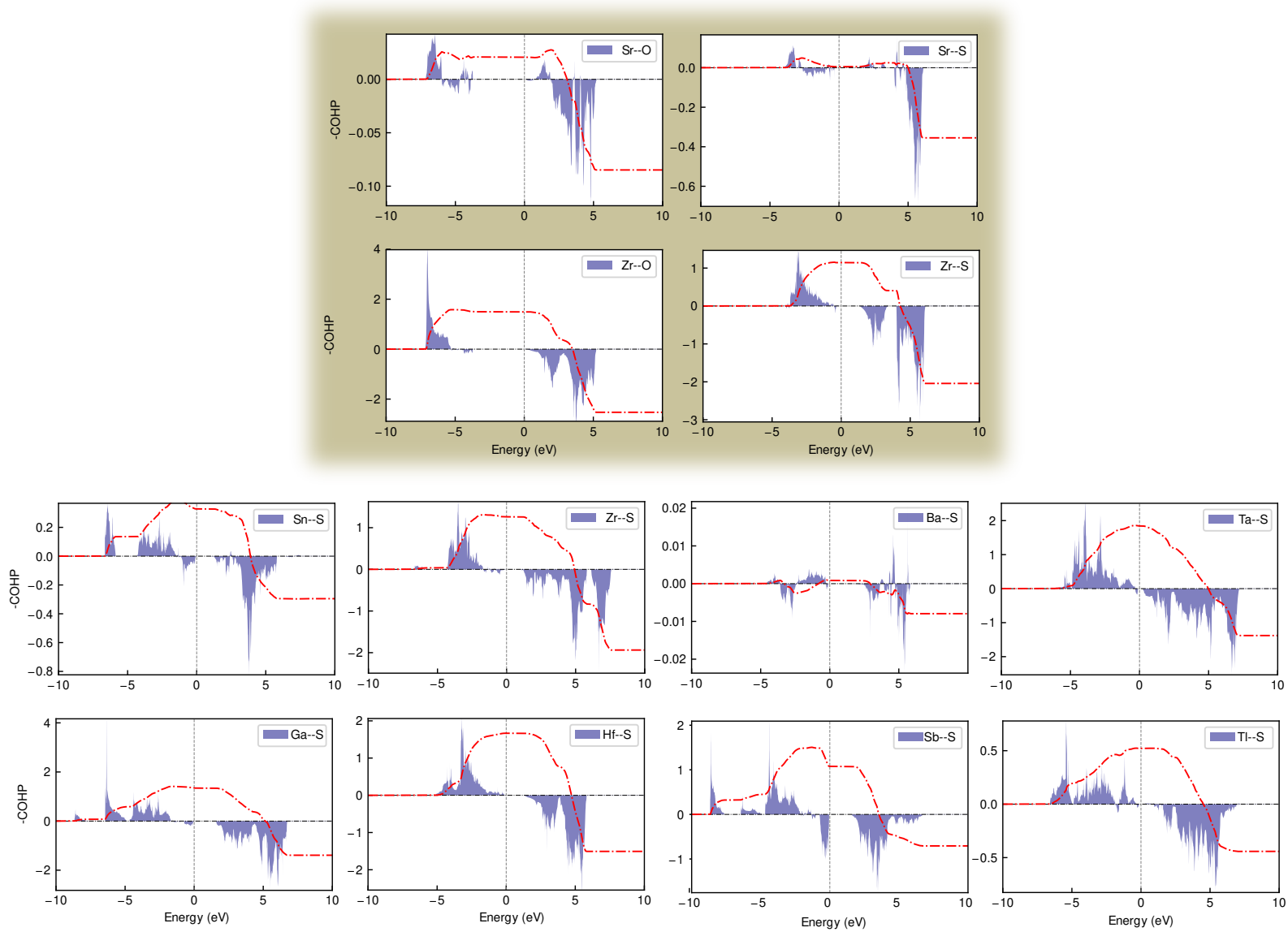

Figure S10: Crystal Orbital Hamilton Population (COHP) analysis. Highlighted part shows the comparison of substitution $\mathrm{S}$ with $\mathrm{O}$ 

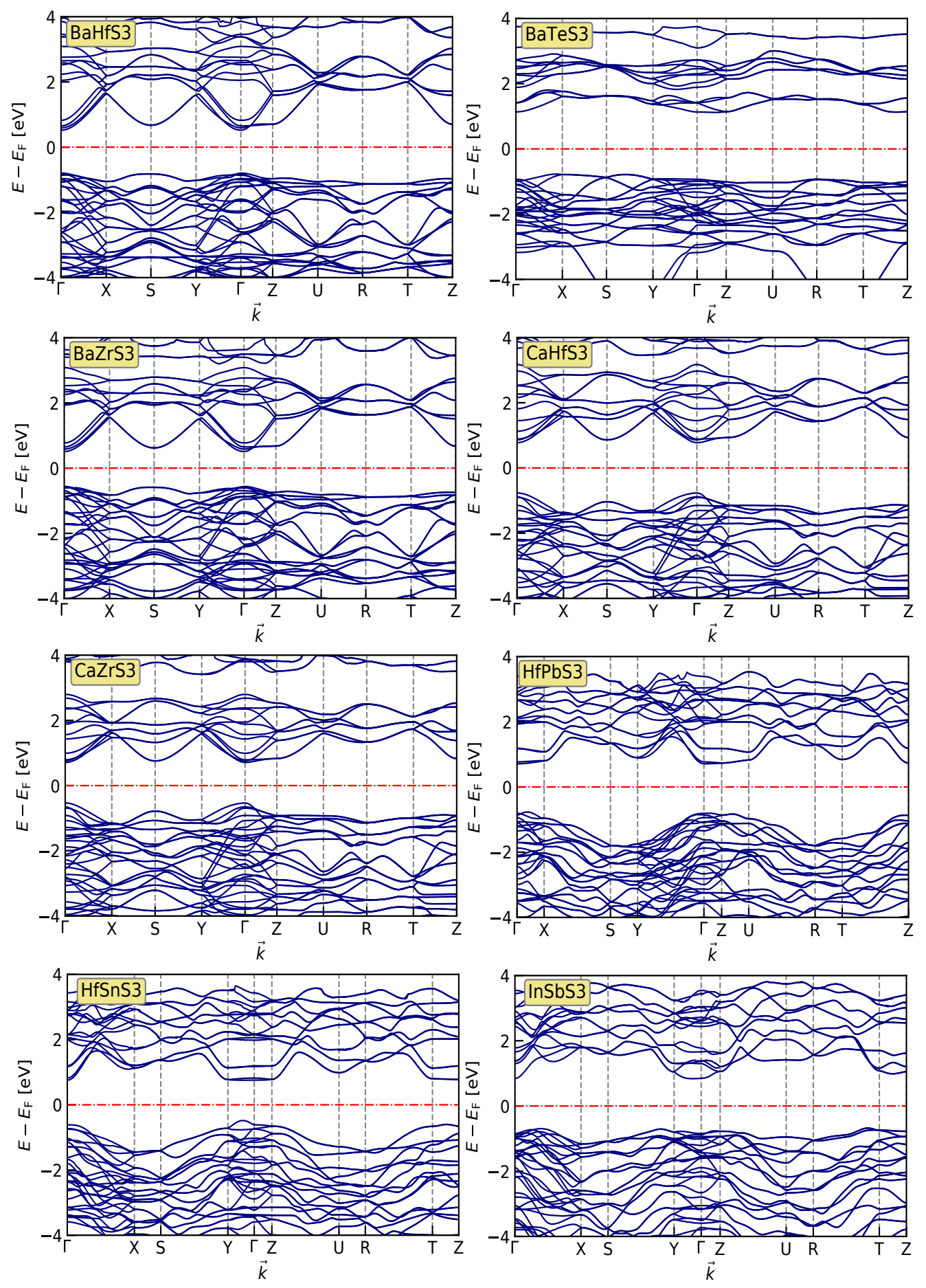

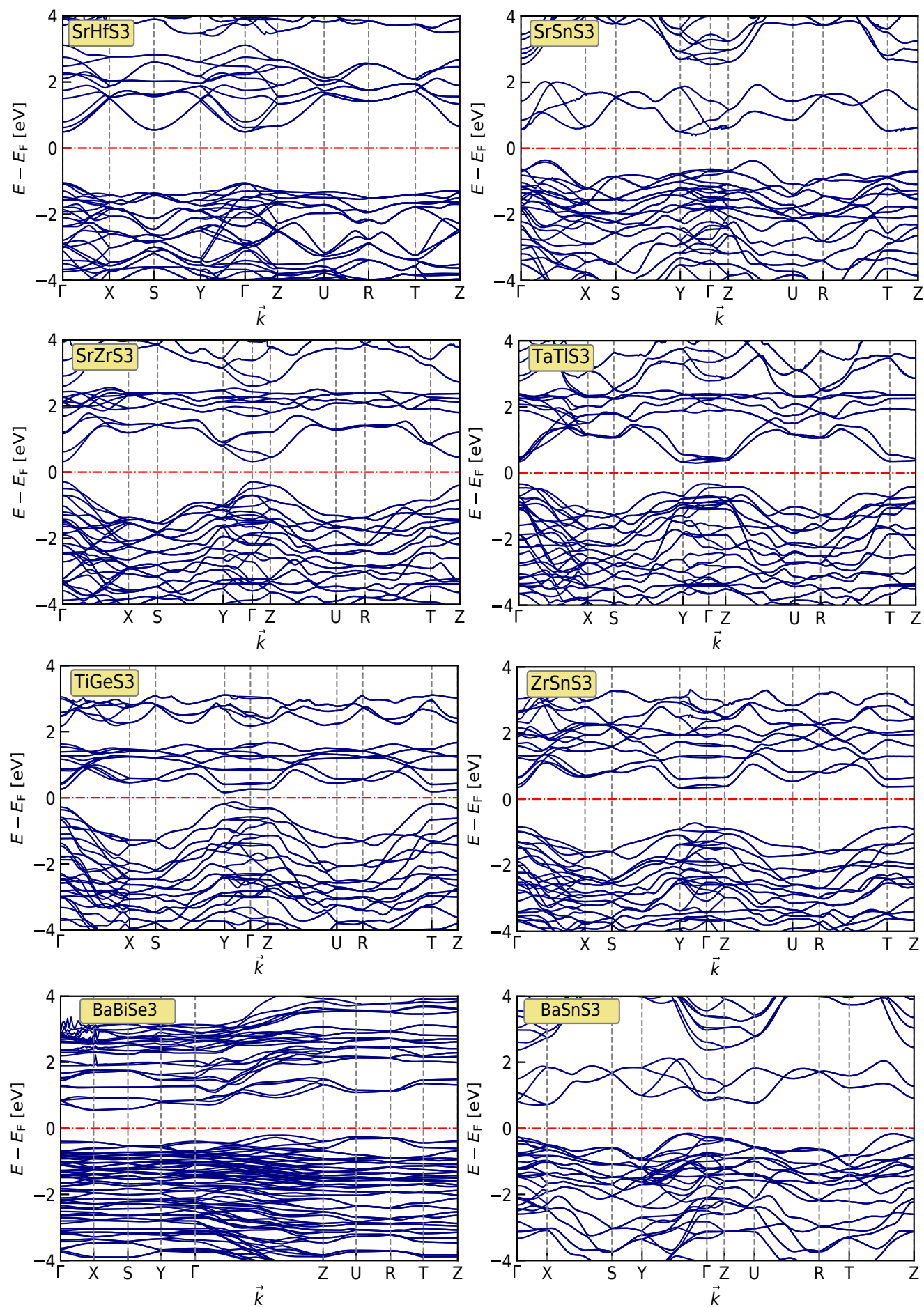

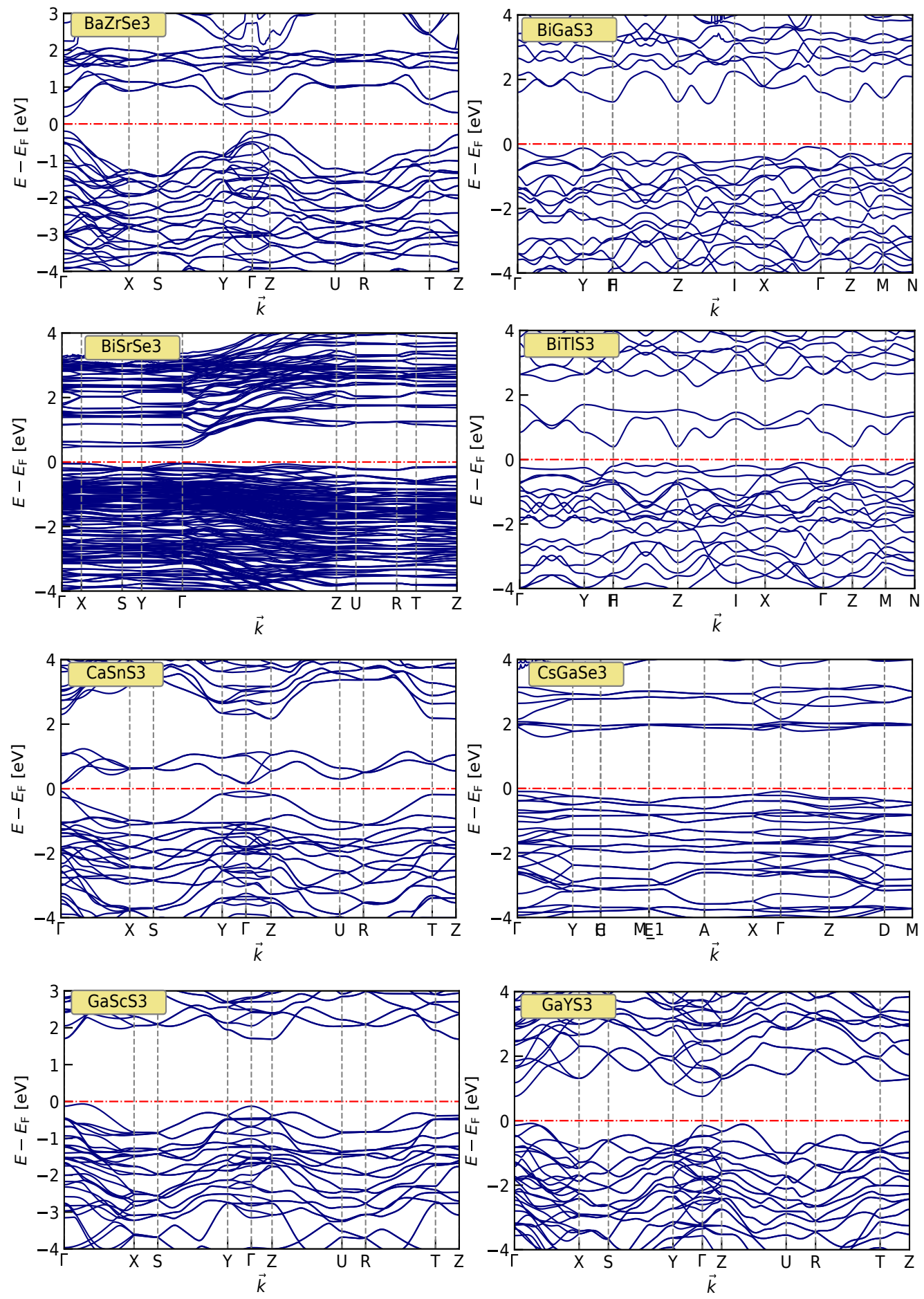

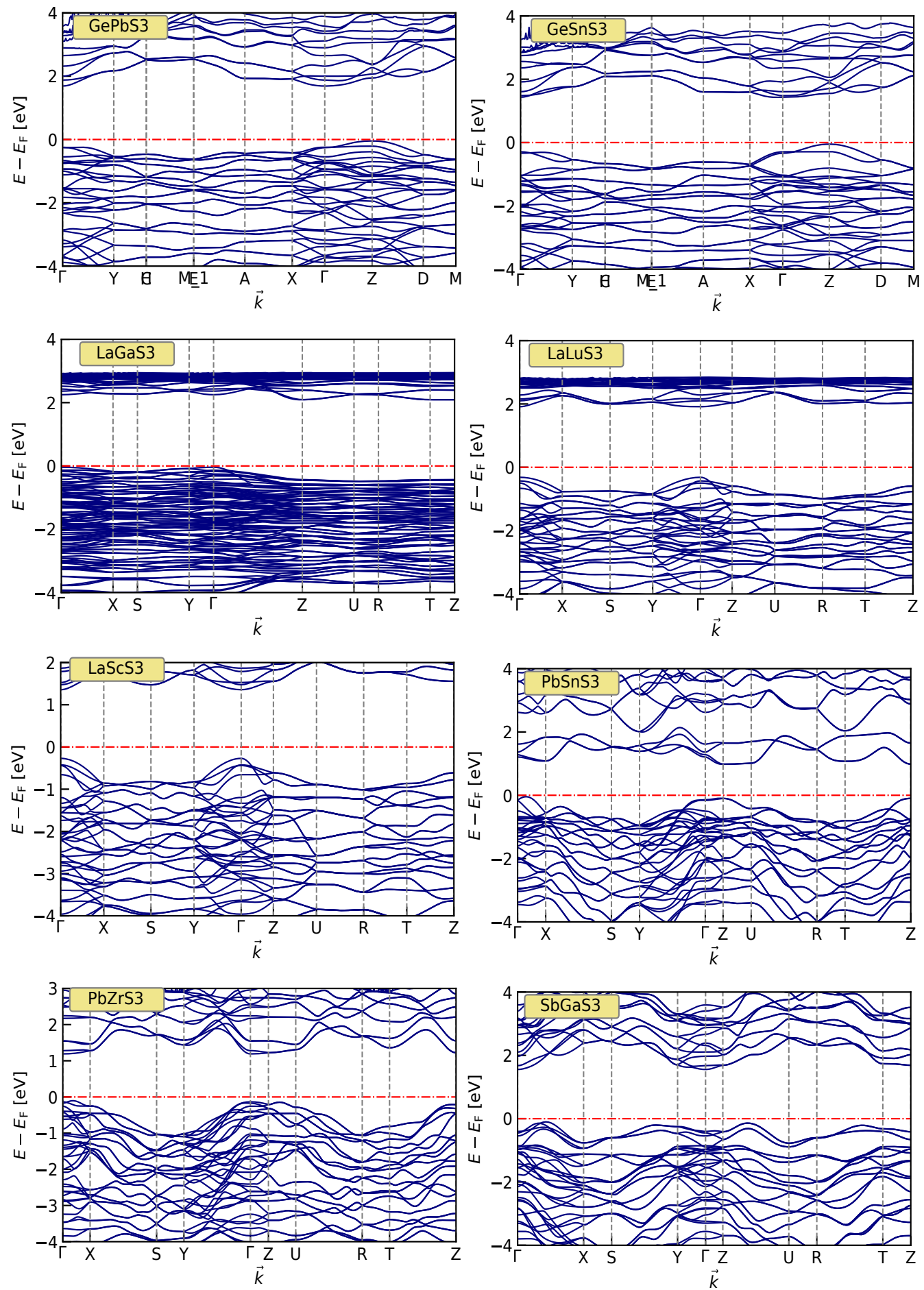

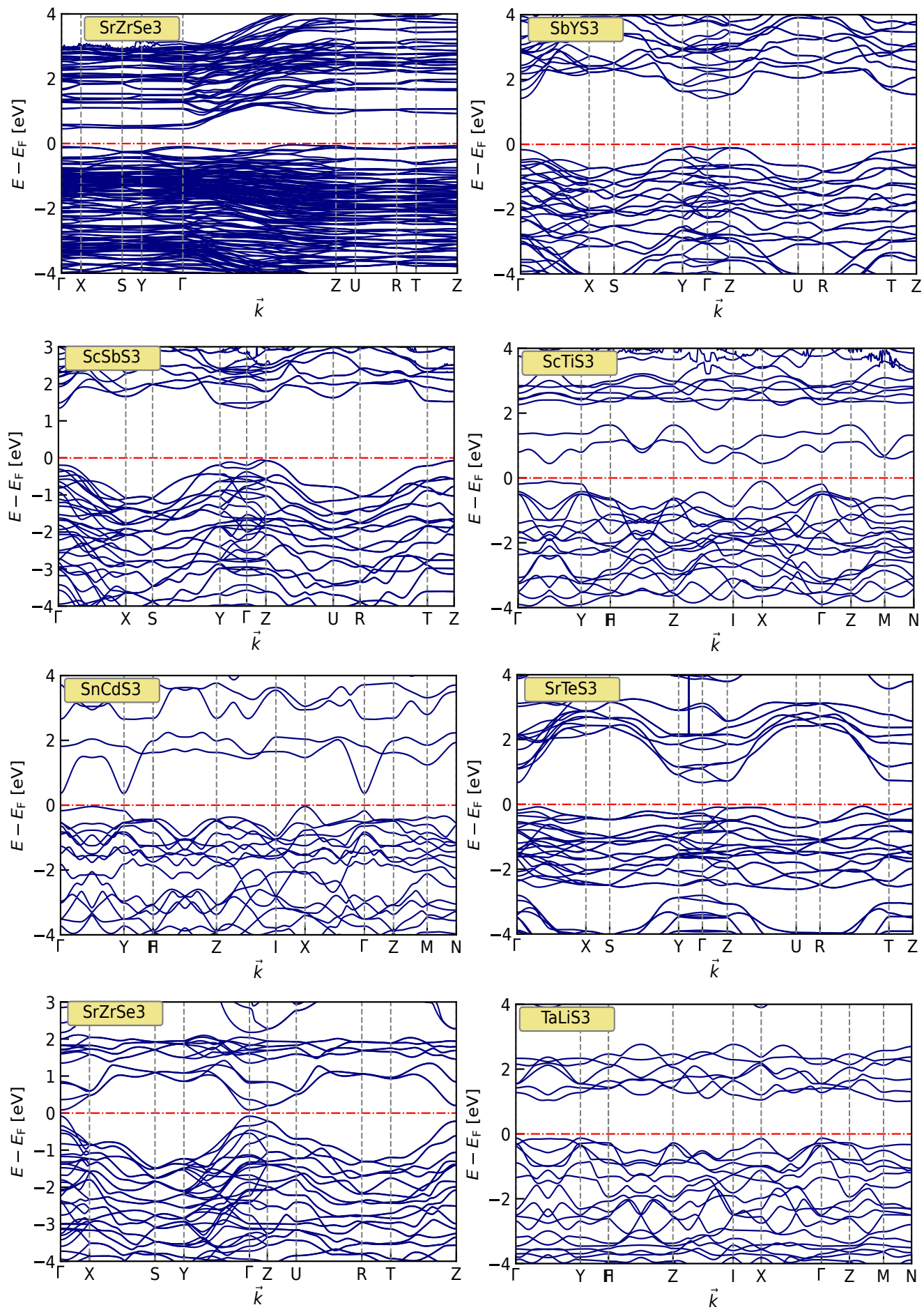

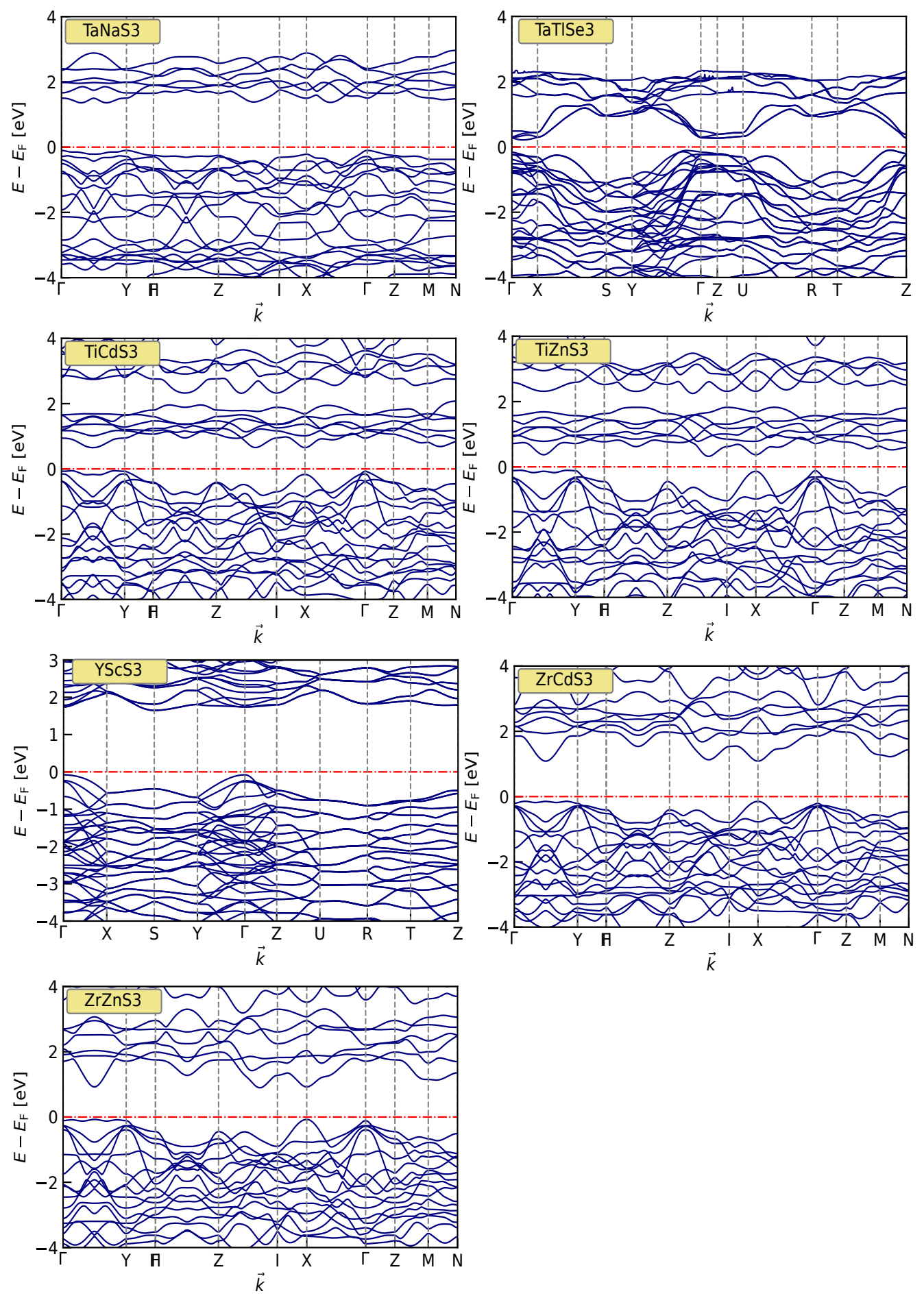

Figure S11: Band structures of the semiconducting materials. 

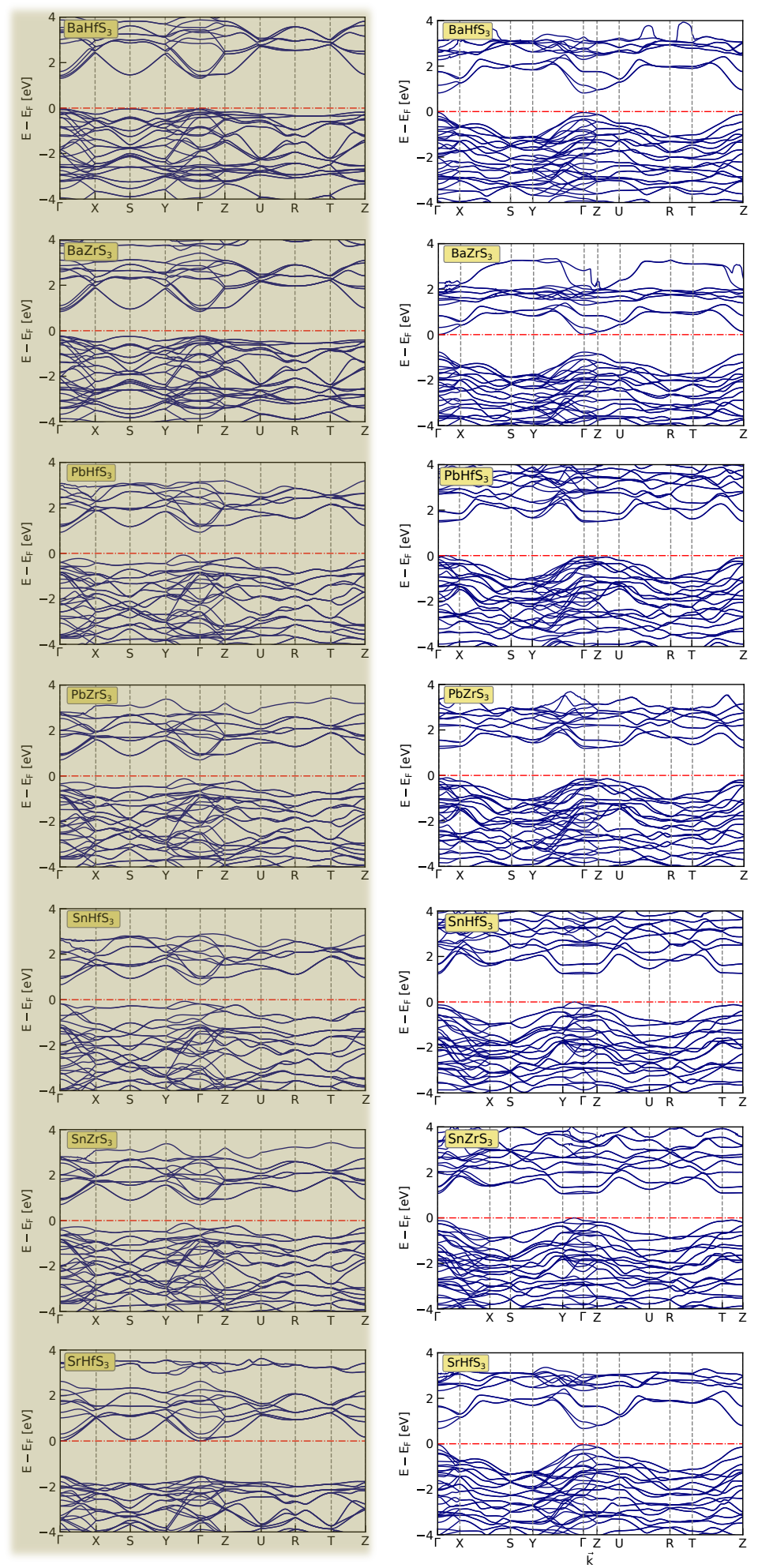

Figure S 12: Calculated Band-Structures for both $\mathrm{Sn}_{2} \mathrm{~S}_{3}$ and $\mathrm{GdFeO}_{3}$ (Highlighted) phases. 

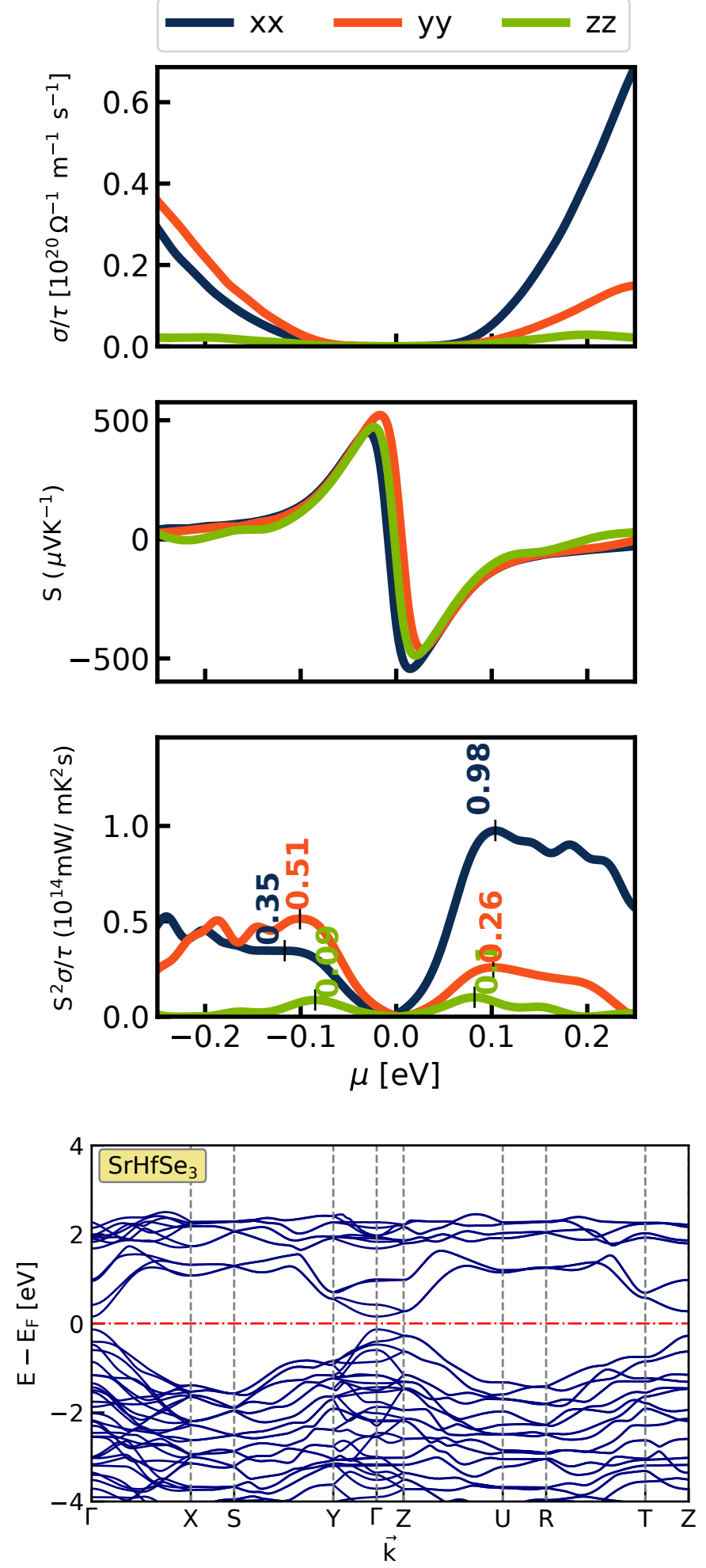

Figure S13: Calculated power factor and Band-Structure for experimental synthesized $\mathrm{SrHfSe}_{3}$ 

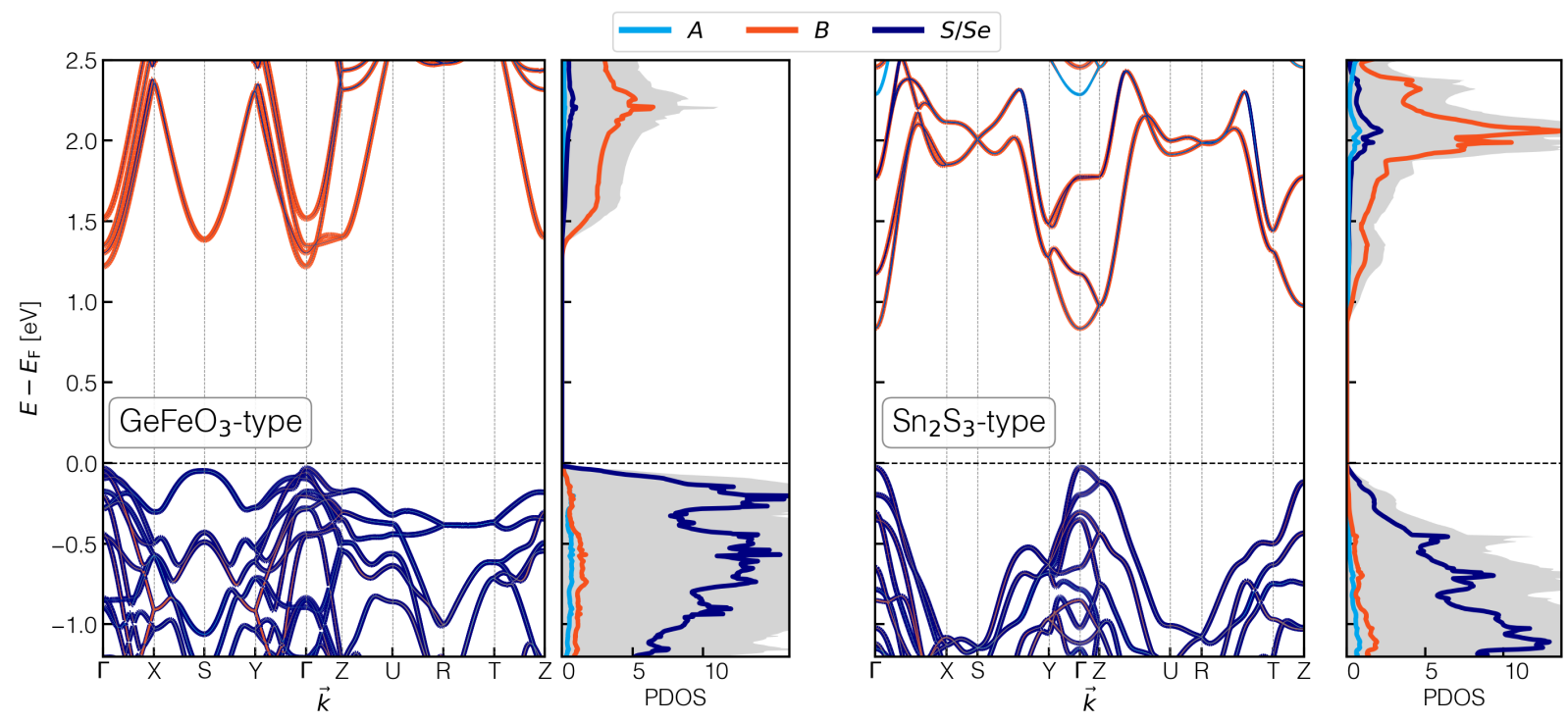

Figure S 14: Comparison of band structures of $\mathrm{BaHfS}_{3}$ in both $\mathrm{GdFeO}_{3}$ and $\mathrm{Sn}_{2} \mathrm{~S}_{3}$ structure types. 